\title{
Non-deterministic kinetic energy within the rotor wakes and boundary layers of low-speed axial fans: Frequency-based decomposition of unforced unsteadiness and turbulence
}

\author{
J.M. FERNÁNDEZ ORO $\dagger^{*},{ }^{*}$, K.M. ARGÜELLES DIAZ†, E. BLANCO MARIGORTA $\dagger$ \\ † Área de Mecánica de Fluidos, Universidad de Oviedo. \\ Campus de Viesques 33271, Gijón (Asturias), Spain
}

\begin{abstract}
A detailed analysis of the non-deterministic scales of the flow in low-speed axial turbomachinery has revealed the presence of significant large-scales unsteadiness, with periodic features different to the blade passing frequency (BPF), which are superimposed to the turbulent structures. Introducing a frequency-based decomposition, this additional component has been segregated from turbulent phenomena so the total unsteadiness has been found to be contributed by three components: forced unsteadiness (deterministic scales), unforced unsteadiness (large-scale unsteadiness) and turbulence (small-scale randomness). Dual hot-wire anemometry has been employed intensively within the stage of a low-speed axial fan to provide a valuable experimental database, where the phaselocked averaging technique has been applied to retrieve time-resolved fluctuations and isolate the non-deterministic contribution. The present investigation shows that the presence of the unforced component is mainly related to instabilities of the rotor wakes and tip vortex structures, as well as wake-wake interactions. Moreover, typical eddies size of this component distribute their energy within the frequency range that is containing an $80 \%$ of the total unsteady kinetic energy. As a consequence, it is expected that LES schemes with accurate spatial discretizations may address this component within the resolved scales of the filter, while unsteady RANS modelling could require additional modelling of the unforced mechanisms. In addition, maps and radial distributions of every component illustrate that major flow patterns are identifiable in all of them, due to the redistribution of all-range scales throughout the energy cascade. Turbulent and forced mechanisms present important variations with the operating conditions, while the unforced component is barely affected by flow rate variations. It is shown that typical values of unforced unsteadiness reach up to a $20 \%$ of the total unsteady energy, even for nominal conditions at midspan of the rotor passage. Higher levels of all the components are found towards tip and hub boundary layers, as the total unsteadiness is reinforced by massive flow separations, tip blockages and major flow disturbances.
\end{abstract}

Keywords: Unsteadiness, unforced scales, turbulence, low-speed axial fan, deterministic, hot-wire anemometry, LES modelling, URANS.

\section{Introduction}

Internal flows in fluid machinery involve a wide range of non-equilibrium phenomena: mixing-out of wakes and boundary layers, inviscid and viscous interactions, diffusion of secondary flows or generation and dissipation of turbulence. These flow structures, inherently unsteady and three-dimensional, are rather diverse with a large number of different length and time scales [1-2]. The varied nature of those physical mechanisms configures a high-complex scenario, with continuous energy fluxes between the unsteady and time-averaged flow patterns [3]. In the literature, the whole spectrum of these all-range fluctuations is known as "total unsteadiness", with a further division discriminating deterministic and non-deterministic structures [4-6]. Typically, deterministic scales are those correlated only with the rotation of the machine (or bladerate frequency), thus including all fluctuations that repeat every revolution and the

\footnotetext{
*Corresponding Author. E-mail: jesusfo@uniovi.es
} 
blade-to-blade variation [7-10]. This means that the deterministic unsteadiness is a wellexpected, repeatable flow feature, particularly concerning wake-core patterns and blade blockages. Also known as "unsteadiness", the deterministic gradients are responsible for the establishment of work transfer between the rotating blades and the fluid [11-13]. The assessment of these periodic events over the time-averaged flow is extremely valuable, deserving major research efforts. Hence, in recent years a number of investigations have been focused towards the understanding of this unsteadiness and its impact on the global performance of both compressors and turbines [14-17]. Nowadays, current trends in design of axial turbomachinery takes into account the averaged influence of unsteady flow sources in the prototype's figures of merit.

On the other hand, non-deterministic scales are mainly composed by turbulent structures. Several authors have investigated the impact of turbulent structures on the boundary layer development [18-20], while others have addressed the effect of freestream turbulence on wake decay and interaction processes [21-22]. Typically, turbulence intensity is described with statistical schemes, after substracting the resolved structures (deterministic flow) from the instantaneous measurements using the phaselocked averaging technique [23-25]. This methodology, well-known in the literature, introduces an averaging (time domain) procedure that considers a unique time scale separating periodic events from random disturbances (the blade passing frequency BPF). Experimental research focused on mean velocity profiles and integral parameters of wakes, as well as turbulence parameters, has employed this technique to study complex turbomachinery flows using diverse measuring techniques: hot-wire, hot-film, LDV, fast-response pressure probes, PIV [26-30]. This exact definition gives valuable results, especially when interest is placed on the phase-averaged flow (periodic events). In the last decade, the fluid dynamic group of the University of Oviedo has realized notable efforts in the analysis and understanding of unsteady flows in axial and centrifugal turbomachines. Particularly, concerning low-speed fans, experimental methodologies have been exploited to provide levels of total unsteadiness and turbulence intensities [31-32]. Recently, Fernández Oro et al. have conducted intensive measurements with hot-wire anemometry in a low-speed axial fan with inlet guide vanes, analyzing the influence of the stator-rotor gap and the operating conditions on the unsteady mechanisms of axial fans. In these studies, complemented also with numerical simulations [33-35], a multi-point traversing system was employed to obtain detailed distributions of the velocity field in several windows covering the passage span for a complete stator pitch. The data set obtained from this facility has been used to investigate many different flow phenomena including wake dispersion and wake transport [35-36], mean flow, turbulent structures and integral length scales [36-37], deterministic stresses [38] and secondary flows and tip vortex in similar fans [39-40]. At present day, as an alternative to the costly experimental campaigns, computational modelling has become an extended, well-mature discipline, especially useful in the design process. High accuracy has been reached in the prediction of mean variables, though the accurate description of turbulent structures is still a problematic issue to deal with. Since Direct Numerical Simulation (DNS) is clearly far beyond practical purposes with current computational resources, two different strategies are considered in the present state-of-the-art: unsteady RANS (URANS) and Very Large Eddy Simulations (VLES), rather than real LES approaches, still unaffordable. It is a common practice that commercial codes implement unsteady RANS simulations, solving time-dependent terms that provide a full-unsteady description on the flow [41-43]. The turbulent stresses derived from the Reynolds-averaging are addressed using a turbulence model, which is generally linked to the strain rates of the resolved velocity field. However, the 
use of full-unsteady codes to simulate the flow within multistage turbomachinery is still a very serious challenge for complex geometries: they require excessively large discretizations and high CPU times and computational resources to achieve a periodic flow solution with acceptable accuracy. Therefore, several authors have worked intensively on this idea, developing a steady methodology which addresses the deterministic sources of unsteadiness through modelling issues [44-47]. As a consequence, a steady simulation can be executed, including both deterministic and Reynolds stresses to take into account the averaged impact of unsteady phenomena and the turbulent generation and dissipation respectively on the time-averaged flow.

Complementarily, approaches based on Large Eddy Simulation (LES) of turbomachinery flows have begun to be considered in the literature. This technique spatially filters the governing equations, using a filter that it is related to the grid density [48-50]: the finer the mesh, the larger the amount of unsteady kinetic energy that can be resolved directly within the filtered set of equations. Typically, depending on the characteristic integral length scale of the problem, a mesh size must be fixed to resolve at least an $80 \%$ of the unsteady kinetic energy (including deterministic fluctuations and turbulent structures). The rest is again modelled using the so-called "subgrid scale" (SGS) turbulence model, typically based on eddy-viscosity formulations which assume isotropic characteristics of the small-scale turbulence.

Summarizing, URANS modelling resolves the deterministic scales and models the nondeterministic structures (mainly, turbulence). In the LES algorithms, both deterministic and most of the non-deterministic structures (the target is approximately an 80\%) are resolved, while inner turbulence (the remaining 20\%) in the inertial subrange is further modelled. Anyway, whatever modelling approach is employed, a validation stage with detailed experimental data is required.

To date it has been assumed that deterministic scales consider periodic events at BPF, while non-deterministic scales are basically composed of turbulence. This is a direct consequence of the unique temporal scale (BPF and harmonics) introduced in the phaseaveraging technique. However, other "large-scale" unsteadiness with periodic features, but unrelated to the shaft speed, can be still present [51]. Recently, the authors have redefined the term "forced unsteadiness" to refer the ensemble-averaged flow clocked with the BPF, which is obtained using the phase-locked averaging technique. Conversely, the "unforced unsteadiness", consisting in "large-scale" mechanisms, includes vortex shedding, unsteadiness of separated points, misalignment of blades or tip vortex instabilities. This kind of large-scale unsteadiness is neither turbulence nor a strictly deterministic contribution, so it is placed somewhere between the deterministic fluctuations and the chaotic turbulence. Since most of the research in the literature has been focused on deterministic unsteadiness or on turbulent structures, unforced unsteadiness is usually ignored, introducing cut-off frequencies or averaging procedures that extract just "small-scale" fluctuations or retrieve exclusively the periodic events. In essence, this means that the total unsteadiness can be decomposed in three parts: turbulence and both forced and unforced unsteadiness, though the difference between them is a very diffuse frontier, due to the complex distribution of energy across the different eddy sizes. Also, deterministic scales are just referred to forced unsteadiness while non-deterministic scales include unforced unsteadiness and turbulence (figure 1). 


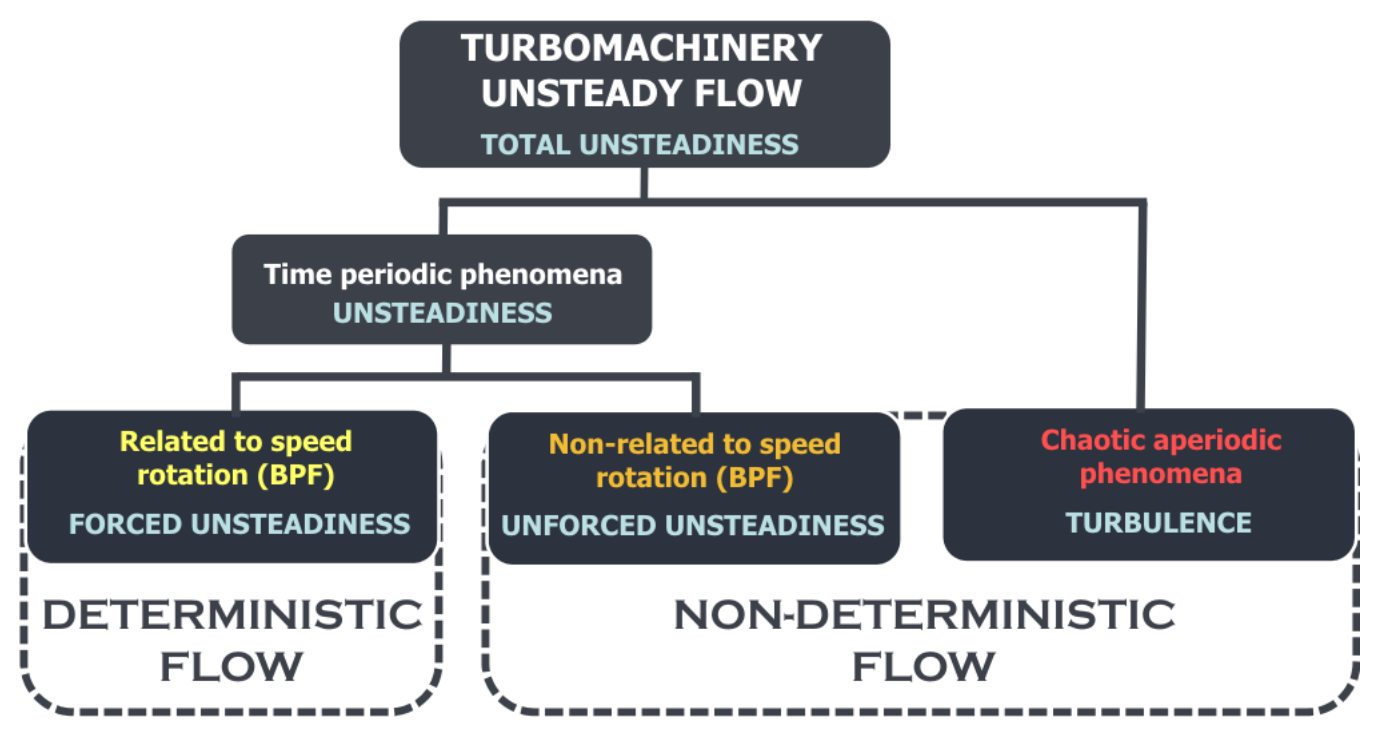

Figure 1. Typology of unsteady processes in turbomachinery flows.

In the present investigation, it is demonstrated that the contribution of the unforced unsteadiness should not be neglected so easily in the complete picture of total unsteadiness. When introducing URANS simulations, this component is completely vanished because only forced sources are resolved and turbulence models are generic, unable to address unforced contributions. On the contrary, in the case of LES algorithms, using sufficiently fine meshes, unforced sources should be captured in the resolution of the filtered equations, because most part of the unforced unsteadiness is distributed over the resolved range of the spectrum (within the $80 \%$ of the total unsteady kinetic energy). Anyway, there is an unforced unsteadiness coming from mechanical instabilities or blade misalignments that can not be predicted even with LES schemes, due to the rigid restrictions of the wall boundaries assumed in CFD techniques.

Most of the turbomachinery URANS simulations published in the literature include a section where numerical results are compared with experimental data for validation. This means that, in the particular analysis of turbulence parameters, depending on the definition of the turbulence levels for both experimental and numerical results, significant discrepancies could be derived from the missing unforced disturbances in the numerical scheme. The following study provides an estimation of the relevance of the unforced unsteadiness in the total unsteady kinetic energy within turbomachinery flows. Also, the influence of the boundary layers and the anisotropy of the rotor wakes are analyzed, searching for additional trends relating unforced unsteadiness and secondary flows. The main objective is to show that additional modelling to take into account unforced fluctuations could be necessary in URANS calculations.

\section{Experimental Setup and Database}

\section{Open-loop facility}

The experimental measurements have been conducted on a single-stage, low-speed axial fan with inlet guide vanes, operated in an open-loop facility that includes a throttle at the inlet to modify the mass flow rate delivered. A Venturi nozzle has been designed 
according to standard normative BS848 and installed upstream of the stage to measure the volumetric flow rate. Figure 2 a shows a sketch of the test facility, with a total length of 20 fan diameters between the throttle and the fan inlet to assure uniform inflow.

The facility has been constructed with the possibility of changing the axial gap between the rows. The stator-rotor gap can be modified using a set of annular rings that can be removed or added in the fan hub and shroud to increase or decrease the interrow distance. Typically, two different axial gaps were tested in the present database: a larger nominal gap of $50 \%$ of the blade chord, and a lower gap of a $37 \%$ of the blade chord, both at the hub section. The rotor is directly coupled to a $37 \mathrm{~kW}$ AC motor which is placed at the turbomachine discharge. The power is supplied by an industrial converter that also fixes the rotational speed of the fan, $2400 \mathrm{rpm}$, with maximum deviations of \pm 2 rpm.

\section{Low-speed axial fan}

The single-stage axial flow fan shown in figure 2-b has a 13-vane stator followed by a 9-blade rotor. The stator vanes are based on the British circular family $\mathrm{C} 1$, with a chord length varying from $157 \mathrm{~mm}$ at the hub to $170.8 \mathrm{~mm}$ at the tip, a span of $220 \mathrm{~mm}$ and a constant thickness of a $3 \%$ respect to the chord. The blades were designed according to baseline coordinates of a NACA 65-012(10), and radially twisted to provide a freevortex distribution of the absolute tangential velocity. The blades chord length goes from $179 \mathrm{~mm}$ at the hub to $157.4 \mathrm{~mm}$ at the tip with a thickness-to-chord ratio also ranging from $12 \%$ to $8 \%$. The Reynolds number based on the tip speed and rotor chord length is $1.4 \times 10^{6}$, and the tip clearance is $2.3 \%$ of the blade height. At nominal conditions, the volumetric flow rate is $16.5 \mathrm{~m}^{3} / \mathrm{s}$ with a total pressure increment of 1150 $\mathrm{Pa}$. More details can be found in [36-37], and the main geometrical parameters are summarized in a table in the figure $2-b$.

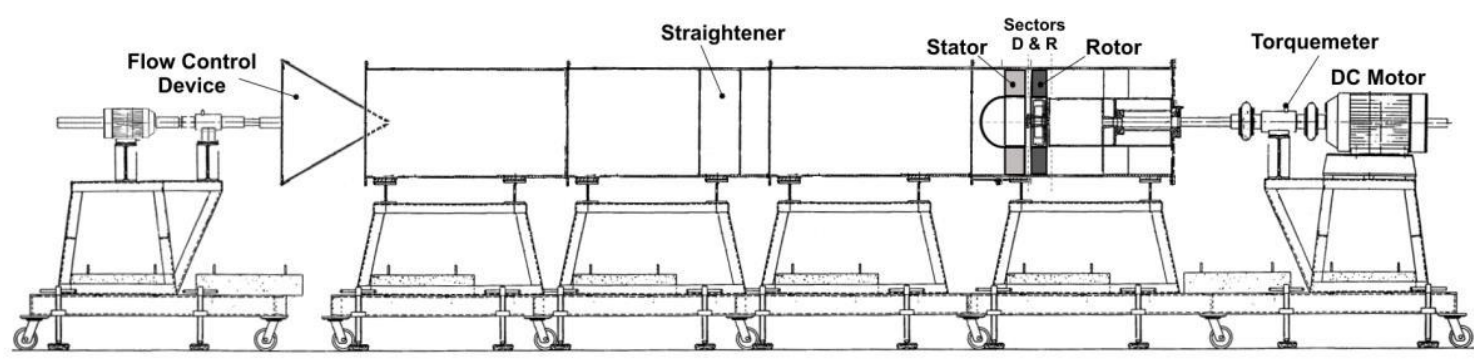

a) Experimental Setup: Open-loop facility

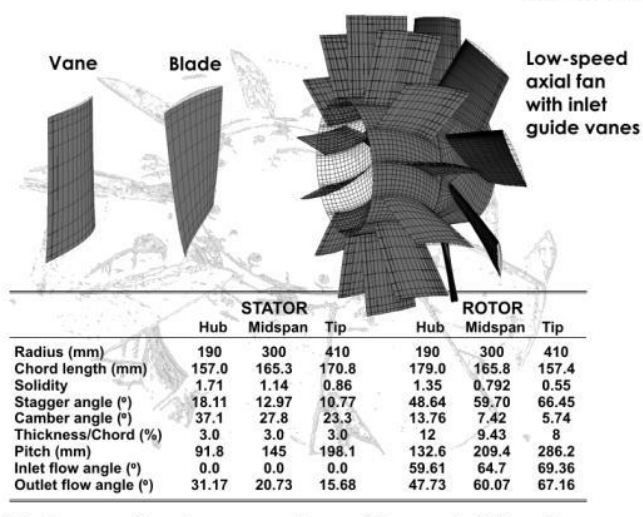

b) Geometrical parameters of the axial flow fan

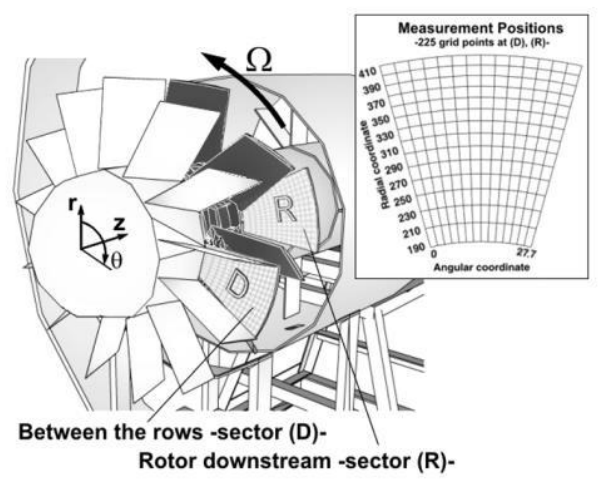

c) Windows in the test section. Experimental data grid of the hot-wire database

Figure 2. Sketch of the test facility. Description of the geometrical parameters of the axial turbomachine. Test sections and data grid on the windows. 


\section{Measuring techniques and database}

The experimental data have been obtained using dual hot-wire anemometry to measure both axial and tangential velocity fields across the stage. The anemometric probe was composed of two tungsten filament wires of $5 \mu \mathrm{m}$ diameter, in a $120 \mathrm{deg}$ crossed layout, with output signals connected to a TSI IFA 100 constant temperature anemometer. The frequency response of the wires was estimated to be about $30 \mathrm{kHz}$, respectively. Maximum angular range was found to be $\pm 50 \mathrm{deg}$, with an uncertainty of the absolute velocity value as high as a $1.3 \%$ in the central zones of the probe angular range and reaching up to $3 \%$ close to the limits.

Since the absence of additional stages allows us to observe all the unsteadiness inside a circular sector with stator pitch periodicity, the measurements have covered the whole span of the machine over just one stator pitch. Two different windows have been considered in the present database, introducing a $15 \times 15$ points grid to obtain an accurate spatial resolution: $15 \times 90 \mathrm{~mm}^{2}$ (figure 2). The first one, denoted as " $\mathrm{D}$ " in the figure, is placed in the interrow region, $15 \mathrm{~mm}$ behind the stator trailing edge. The other one, denoted as "R", was defined at the rotor exit, $50 \mathrm{~mm}$ downstream of the rotor trailing edge.

Additionally to the two axial gaps considered, three different operating conditions have been studied in these investigations. The three operating points have been denoted as "nominal", $\phi=0.38$, "partial load", $\phi=0.32$, and "near stall", $\phi=0.27$, conditions, where $\phi$ stands for the flow coefficient defined as the ratio between the overall mean velocity for each test and the blade tip velocity.

In the last two decades, the phase-locked averaging technique has been widely used in combination with hot-wire anemometry to study the flow inside axial turbomachinery. To segregate between deterministic and non-deterministic scales, it is necessary to pay particular attention to the number of samples adopted per blade passing period, as well as the total number of samples considered for an accurate statistical reduction of the velocity traces.

The number of samples for each blade passing period gives a good idea of the circumferential precision of the measurements. This value is related to both acquiring (sampling frequency) and blade passing frequencies (BPF), and also to the number of rotor blades. An accurate value is essential to obtain a precise description of the gradients associated to the wake shear layers when measuring with stationary probes. In the literature, the tangential discretization is chosen to be in the order of one point per degree, resulting in typical ranges between 50 and 100 samples per blade passing period.

On the other hand, the total number of samples depends only on the length of the acquiring time. That is, more samples are stored if the acquiring time is increased. Unfortunately, this time cannot be indefinitely extended because the store space or the duration of the tests is limited. As a result, a moderate number of samples must be adopted, but enough to provide a converged description of the time-resolved flow structures. Typically, the total number of samples to be used in the ensemble-averaging process ranges from 100 to 300 [52], with a disparity that depends on the levels of turbulence, secondary flows and the global disturbance of the flow field.

In the present investigation, a temporal resolution of 100 samples per blade passing period has been fixed, resulting in a sampling rate of $36 \mathrm{kHz}$. Also, for every velocity trace, 100 blade passing periods were recorded approximately, so a total number of $10^{4}$ samples were stored during $0.277 \mathrm{~s}$ at every measuring location. As a result, for the entire database, comprising 2 axial gaps and 3 operating conditions, 1200 maps of time- 
resolved velocities were retrieved. Figure 3 illustrates the degree of convergence achieved with this election. The convergence of the phase-averaged data is presented only for the axial velocity component, which is more restrictive than the tangential one (axial velocity presents higher gradients and fluctuations than the tangential component [36]). The results are presented for two representative points, one placed at midspan and the other in the hub boundary layer (see the dashed lines in the time-averaged contour plot), for both nominal and near stall conditions. The time-resolved evolution of both traces at nominal conditions, obtained averaging 100 total samples in each of the 100 points that sampled the blade passing period $\left(T_{R}\right)$, is also added in the figure. The convergence in the total number of samples adopted to obtain the time-resolved structures is analyzed introducing the residual $R$ as the rms value of the difference between the time-resolved traces using $m$ and $m-1$ samples and divided by the averaged value of the trace as a scaling factor. This scaled residual, a more appropriate indicator of convergence, is formulated as follows:

$$
R=\frac{\sqrt{M \sum_{m=1}^{M}\left(\left(\theta_{l}\right)_{m}^{(k)}-\left(\theta_{l}\right)_{m}^{(k-1)}\right)^{2}}}{\sum_{m=1}^{M}\left(\theta_{l}\right)_{m}^{(k)}}
$$
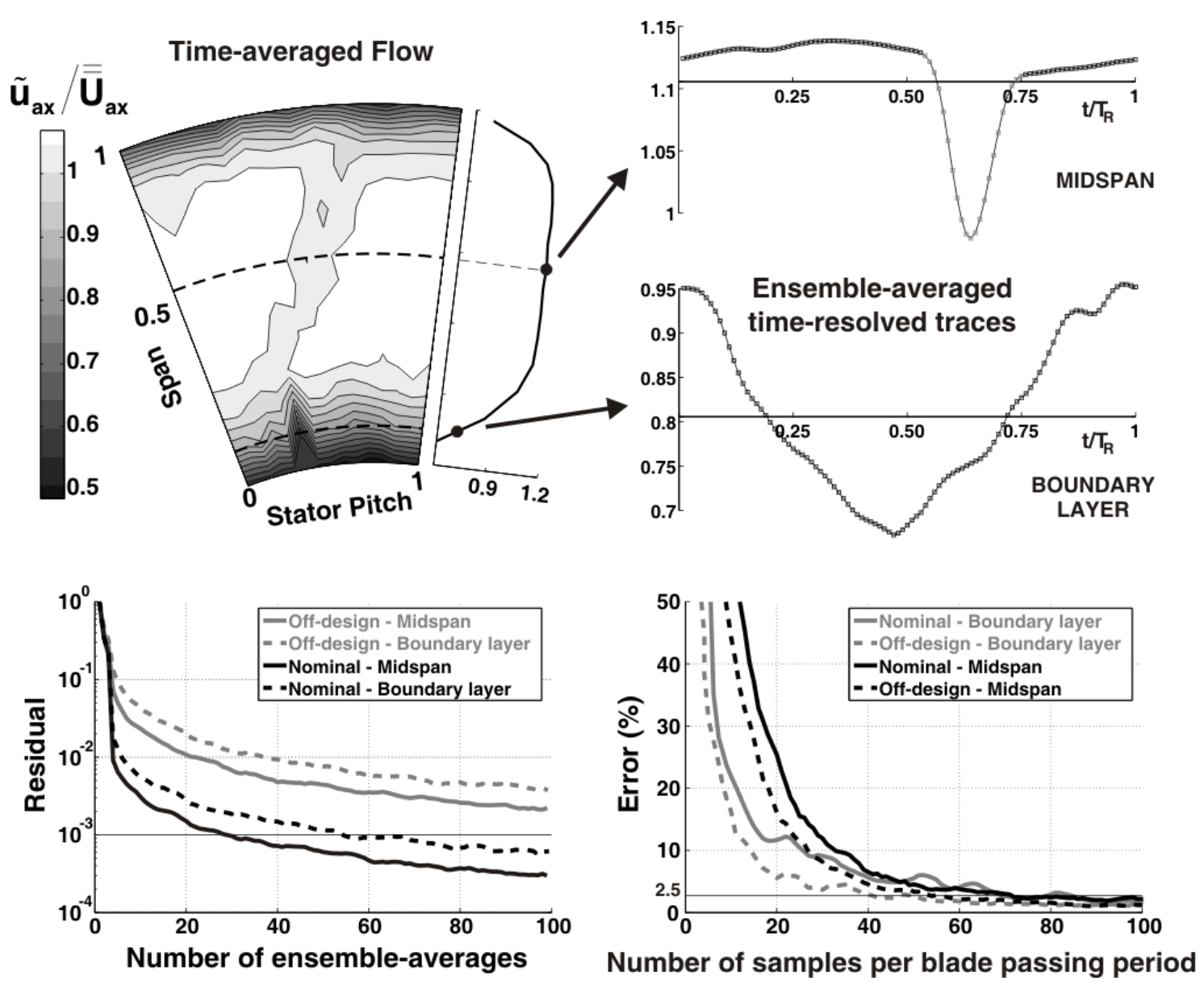

Figure 3. Convergence histories of the axial velocity component measured using dual hot-wire anemometry. Analysis of the number of ensemble-averages and samples per blade passing periods required to obtain stable statistical description of time-resolved structures in inner and outer regions of the blades passage in a low-speed axial fan. 
The results obtained are shown in a logarithmically-scaled plot at the bottom of the figure. The convergence has been evaluated for a number of ensemble averages ranging from 2 to 100 , with a default convergence criterion of $10^{-3}$. Convergence is perfectly met at nominal conditions for both locations when using at least 50-60 ensembleaverages. As expected, a higher number of samples are required within the boundary layers (dashed black line) due to the presence of secondary flows and higher turbulence levels [37]. At off-design conditions, the overall flow disorder affects significantly the spanwise convergence, compromising the fixed criterion. Anyway, the global trend is well-established and the minimum threshold at off-design conditions $\left(1.5 \times 10^{-2}\right.$ in the grey lines) is supposed to be sufficient for the adopted $M=100$ samples.

Additionally, the error associated to a rough description of the ensemble trace for a blade passing period when few samples are used is shown in the bottom plot at the right. This plot represents the difference in the estimation of the energy (the area under the velocity fluctuation) between the time-resolved traces obtained using $n$ and $n-1$ discrete samples. Such differences, that are progressively reduced as the number of samples is increased, are lower than a $2.5 \%$ for all the cases when 80 points are used to describe the blade passing period. It is interesting to note that nominal conditions at midspan need more points to converge than off-design conditions in the boundary layers: offdesign flow patterns involve thicker wakes with lower velocity deficits [37], traceable with less samples than narrow and peaked wakes of nominal operation. The election of $N=100$ points to cover the blade displacement implies that both pressure and suction sides of the rotor wakes have been sampled with at least 10 points, in the order of typical discretizations employed in the literature [53].

\section{Data processing techniques}

The hot-wire traces have been processed in order to segregate deterministic fluctuations from non-deterministic unsteadiness. The deterministic velocity is obtained by ensemble-averaging (or phase-averaging) the 100 blade passages recorded for every velocity trace:

$$
\operatorname{\theta g}(\stackrel{\mathrm{r}}{r}, t)=\left.\frac{1}{M} \sum_{m=1}^{M} u_{i}(r, \tau)\right|_{m} ; \quad \tau=t+\frac{2 \pi}{\Omega B}(m-1)
$$

where the variable ${ }^{1} r$ represents the spatial coordinates and $t$ is the time for a rotor blade passing period (i.e., phase angle of the rotor blades), $B$ is the number of blades and $\tau$ computes for the total acquisition time. Obviously, time and rotor phase are interchangeable variables introducing the machine rotational speed. The nondeterministic scales are directly obtained subtracting the time-resolved (or phaseresolved) velocity for the original, instantaneous trace:

$$
u_{i}^{\prime}(\stackrel{r}{r}, \tau)=u_{i}(\stackrel{r}{r}, \tau)-Q_{i}(\stackrel{r}{r}, t)
$$

The non-deterministic component obtained in (3) results in a raw signal (depending on $\tau$ ) that needs further processing to provide a statistical meaning. Hence, the level of non-deterministic unsteadiness can be estimated applying also the ensemble-averaging: 


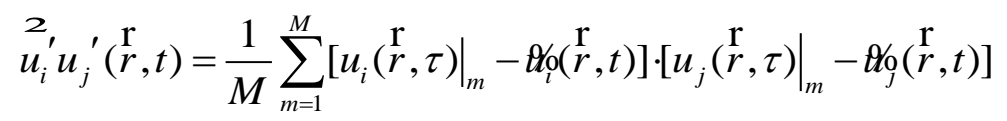

where the phase dependency (i.e., non-deterministic stresses as a function of the time for a rotor blade passing period, $t$ ) reveals the transport of the ensemble, non-resolved structures in the unsteady, deterministic flow patterns.

\section{Non-deterministic scales of the flow}

After subtracting the phase-averaged component of the velocity-eq. (3)-, it is expected that the remaining term contains only the turbulent fluctuations. However, in the vicinity of a rotor wake, other "large-scale" unsteadiness with periodic features unrelated to the shaft speed could be still present $[51,54]$. This means that the timeunresolved fluctuation is mainly composed by turbulent structures, but with additional periodic contributors superimposed to the baseline spectrum of turbulence. This largescale mechanisms, redefined by the authors as "unforced unsteadiness" [40], include vortex shedding, tip vortex instabilities (like an unsteadiness on the unsteadiness), unsteadiness of separated points, rotating stall and rotating instabilities [55], flutter and blade misalignments.

Previous investigations have analyzed in detail the flow structures of the deterministic fluctuations [36-37], especially concerning rotor-stator interaction in the axial gap [38]. A detailed picture of the unsteady deterministic flow and the turbulence levels was already highlighted in the interrow region. Now, we focus on the contribution of the unforced scales at the rotor discharge, with major interest on the relative importance of its energy content.

To obtain the unforced component, it is necessary to introduce a filtering procedure to isolate the discrete peaks immersed in the turbulent scales of the time-unresolved fluctuation. As a starting point, the deterministic scales must be removed from the total unsteadiness to retrieve only non-deterministic contributions. In figure 4 , the periodogram (or discrete fourier transform - DFT) of a raw velocity trace (named in the figure as "instantaneous") has been represented to illustrate the decomposition. Introducing the phase-locked averaging technique, all those peaks corresponding to the BPF and harmonics are segregated in the time domain (subplot "deterministic"). In the frequential space, the ensemble-averaged component shows no contributions of largescales below the BPF and just higher harmonics appear to configure the particular shape of the blade-to-blade velocity gradient. Finally, subtracting the ensemble-averaged velocity from the raw signal, the non-deterministic scales are obtained in the bottom subplot. In black lines, all the figures represent the periodogram of the different velocity structures, which is a rough estimate of the power spectrum. The mathematical restrictions associated to the FFT algorithm, like the assumption of decomposition in a linear series of sine waves, the limited number of total points sampled per trace, the window function employed to minimize the leakage in spectral estimates or the assumption of stationary signals, limits its reliability and leads to the appearance of notable experimental scatter in the spectrum. In logarithmic plots, the presence of spurious spikes reveals the variance of the periodogram, which can be affected by a random inaccuracy of the order of $100 \%$ [56]. It is well-known that the performance of the FFT algorithm can be improved using an ensemble of estimates for a number of different subrecords [52], i.e. the Welch method, though the random noise cannot be fully removed [57]. 

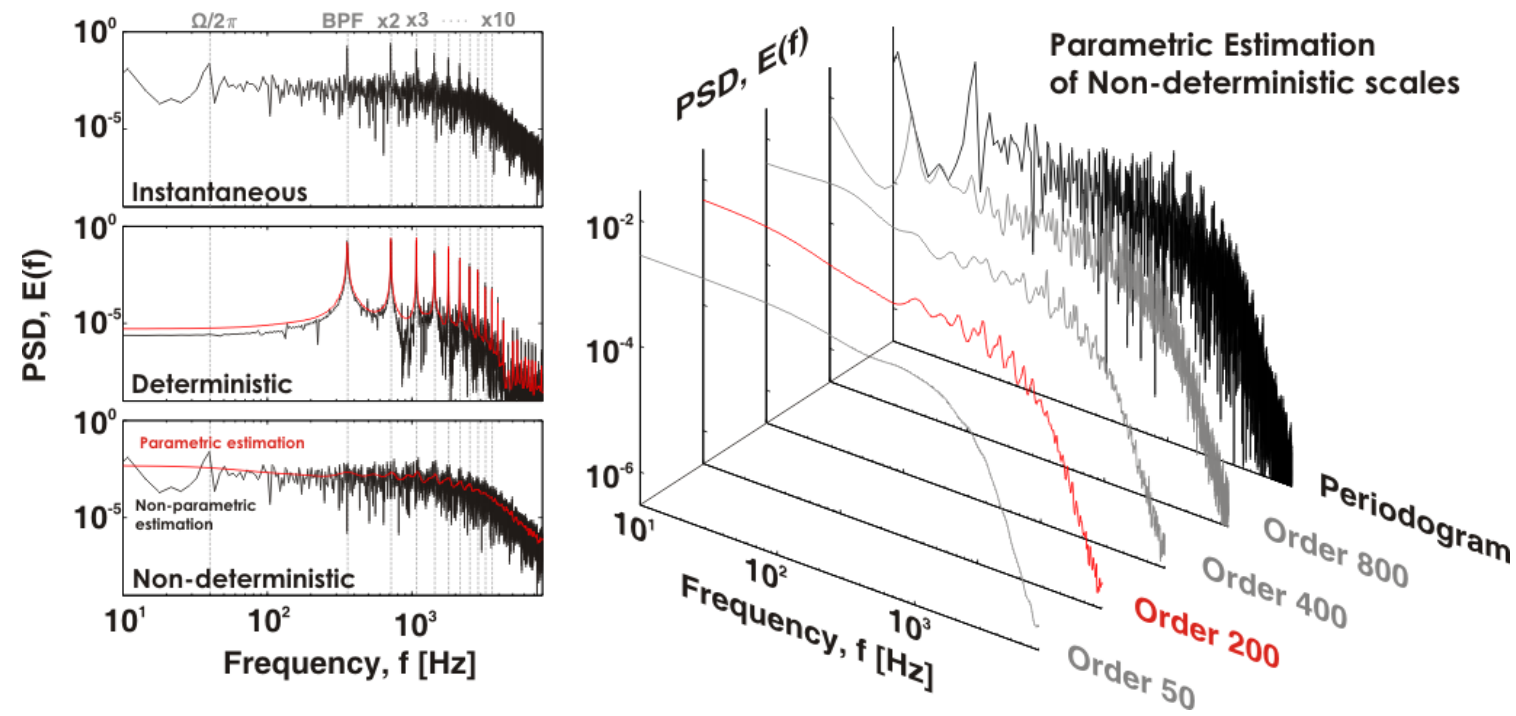

Figure 4. Decomposition of instantaneous traces in deterministic and non-deterministic components. Parametric estimation of non-deterministic scales to isolate unforced events from baseline spectrum of turbulence.

Alternatively, an estimation of the baseline spectrum can be obtained introducing a smoothed periodogram based on a parametric algorithm. Among the various possibilities, the Yule-Walker AR method has been employed in the present investigation, which fits an autoregressive (AR) model to the windowed input data by minimizing the forward prediction error in the least squares sense. The estimation order of the autoregressive is of central importance because as the periodogram is smoothed more and more, the estimated spectrum approaches to a featureless curve; if the order is too large, the spurious peaks in the spectrum are not removed; whereas if the order is too small, the unforced events are smeared out. This is illustrated in the right plot of figure 4, where a progressive order reduction has been applied on the parametric estimation of the non-deterministic scales. Unfortunately, the smoothness degree that it is necessary to eliminate spurious noise while maintaining the relevant peaks cannot be determined easily. Typically, theory of statistical analysis reports of the final prediction error as an approach to derive the optimum order, but it has been found that this criterion is not reliable for unsteady phenomena in turbomachinery [58]. This seems to be a consequence of the distribution of energy across the different eddy sizes: every eddy size (i.e., temporal scale) is really contributing across the full range of wave numbers, with a hump distribution that is locally centered on the particular frequency of interest [59]. Therefore, the unforced peaks superimposed to the turbulence spectrum reveal the existence of a periodic event, but as we move forward to the extremes of the hump, the energy budget turns to be of the small-scale turbulence type. Hence, an excessively large order of the autoregressive model implies the presence of smoothed peaks, due to an unphysical energy transfer from unforced unsteadiness to the turbulent scales. In other words, an inappropriate election of the autoregressive order leads to an overestimation of the turbulent kinetic energy. In the present investigation, a typical autoregressive order of 200 has been considered as a representative value to preserve unforced peaks within the baseline spectrum of turbulence. With this election, the randomness in the estimated spectrum was completely removed in the large-scale region (up to $80 \%$ of the total non-deterministic kinetic energy). A similar value was used by Barmpalias et al. [58] to analyze unsteady signals in the tip cavities of axial turbines. They used data sampled at $200 \mathrm{kHz}$ for time signals with a BPF of $1.84 \mathrm{kHz}$, resulting 
in approximately 108 points per blade passing period. The similarity in the number of samples per rotor periodicity with the present case justifies the same election of the order of our model. Finally, the parametric estimation of both deterministic and nondeterministic scales has been represented in red in the left subplots of figure 4. As expected, for the deterministic component, both parametric and non-parametric methods provide similar estimations of the fundamental peaks, corroborating that the variance of the periodogram is negligible at the discrete contributions. Only differences are observed at high frequencies where the energy content is extremely low. Regarding the non-deterministic estimation, the baseline spectrum preserves the universal power-law decay in the inertial subrange (classical $-5 / 3$ exponential decay of nearly isotropic, fullydeveloped turbulence), with a superimposed series of bumps showing high-order periodicities not removed by the ensemble averaging. With no scatter in the representation, it is assumed that all those peaks constitute the energy budget associated to the unforced unsteadiness.

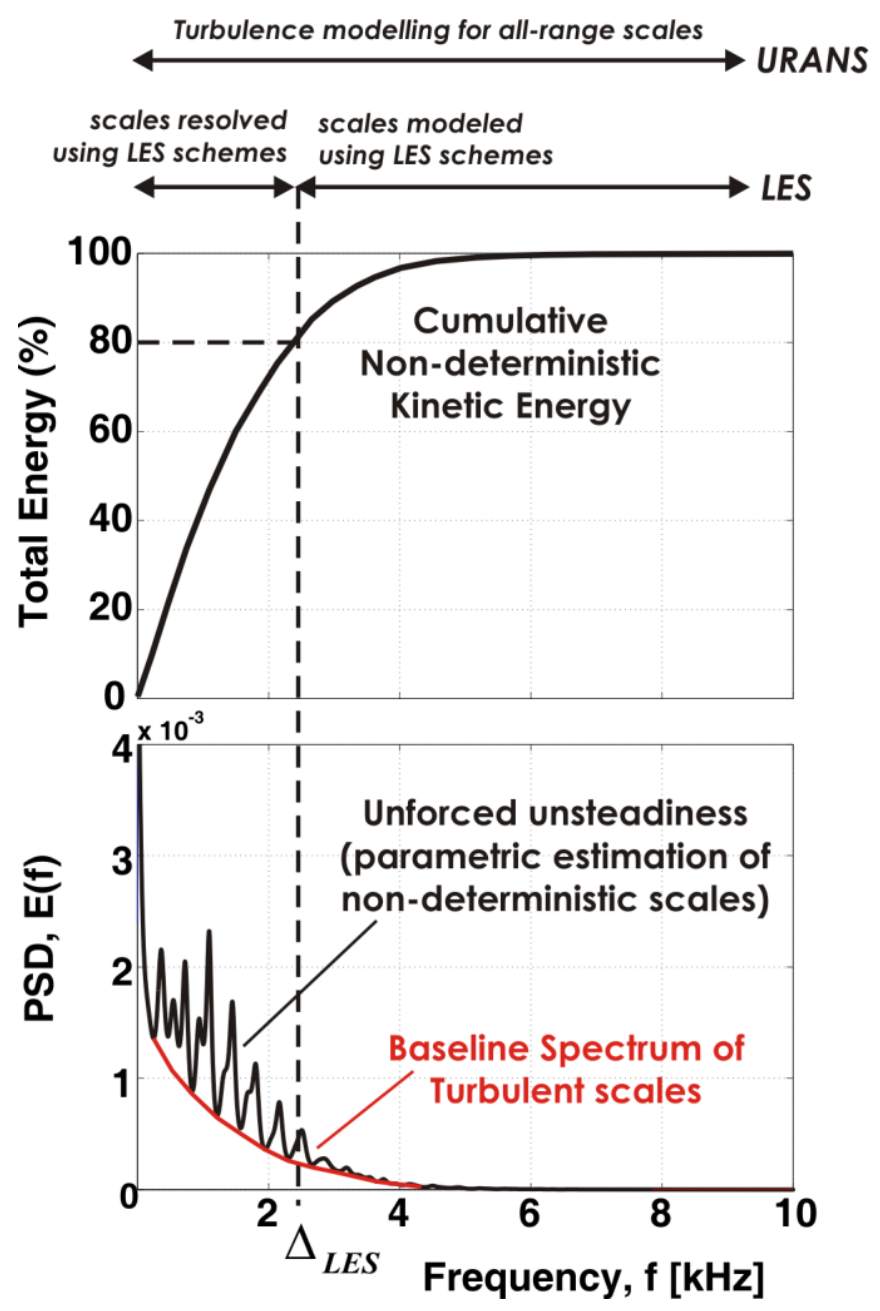

Figure 5. Energy content and frequency distribution of unforced scales over baseline spectrum of turbulent scales.

Figure 5 shows in a linear plot the unforced component retrieved using the parametric estimation of the power spectrum density. The background level, marked with a red line, represents the energy distribution of the turbulent scales. It has been obtained using an iterating procedure to identify the local minimum values of the unforced peaks. 
Afterwards, these minima are fitted to a $-5 / 3$ power law decay in the inertial sub-range (approx. from $1 \mathrm{kHz}$ on) in order to obtain a continuous evolution. The integration of the area under the red curve provides the estimation of the turbulent kinetic energy throughout the whole range of the spectrum. In the same fashion, the area under the unforced peaks until the red line corresponds to the energy content of the unforced scales of the flow. Obviously, the non-deterministic kinetic energy is obtained considering both components, the whole area underneath the black line, according to:

$$
\frac{1}{2}\left\langle u^{\prime 2}\right\rangle=\int_{0}^{\infty} E(f) d f
$$

Additionally, the cumulative kinetic energy of the non-deterministic scales has been represented on top of the figure. Expressed in terms of percentage respect to the total value -obtained in (5)-, this representation provides valuable information about the frequency interval for which a determined amount of energy is contained. Typically, for most turbulent flows, an inferior threshold of an $80 \%$ of the total energy constitutes a practical limit to take into account the relevant range of the turbulent scales. Moreover, major part of numerical simulations using LES schemes employs the frequency value corresponding to an $80 \%$ of cumulative turbulent kinetic energy to fit the mesh filter size, $\Delta_{L E S}[49,60]$. This implies that far beyond that frequency, the remaining energy content is modelled, whereas the previous range is resolved within the filtered equations. In the present database, it is confirmed that most of the unforced contributions are placed within the $80 \%$ energy range, pointing out the accuracy of LES techniques to resolve unforced mechanisms without further modelling.

\section{Results and discussion}

On the estimation of accurate mesh sizes to solve LES modelling

A comprehensive description of the main flow structures and turbulence levels has been already discussed in previous investigations for the present database [36-38]. In those papers, the unforced unsteadiness had been removed from the turbulent structures using a filtering procedure to eliminate high-order periodicities [37]. Now, we are revisiting the non-deterministic contributions to analyze the importance of the kinetic energy associated to the unforced component.

As a first approach, an analysis of the cut-off frequency $-\mathrm{f}_{80 \%-}$ which implies that an $80 \%$ of the non-deterministic energy is contained within such frequency range is carried out. This alternative metric gives a precise idea of the typical eddy sizes of the nondeterministic scales involved in low-speed axial turbomachinery. To that end, a composite picture is shown in figure 6, introducing maps of $\mathrm{f}_{80 \%}$ in the interrow spacing and rotor downstream for the axial fan working at nominal conditions. Most of the flow structures developed within the stage are perfectly identifiable in the distributions. Between the rows, the stator wake (marked with a dashed line) is characterized with a cut-off frequency of $2500 \mathrm{~Hz}$, revealing the generation of small-scale turbulence within the shear layers. In addition, towards the suction side of the vanes, a turbulent spot indicates the presence of separated flow induced by the interference of the rotor blockage. This mechanism, already analysed in [36] in the case of the lowest axial gap, results from the aerodynamic loadings of the suction sides which overturn the flow streamlines. A background level between 500 and $800 \mathrm{~Hz}$ in the inner regions shows the 
largest scales of turbulence, characteristic of the inviscid flow passing through the stator passage at midspan locations. In the tip region, the frequency raises up to $1700 \mathrm{~Hz}$ due to the generation of turbulence in the boundary layer. Close to the hub, the boundary layer is still not fully-developed, as a consequence of the proximity of the semispherical nose fairing (see fig. 2), so the frequency limit is moderate. Notice that in the interrow spacing, the unforced contributors are practically negligible, since only potential interaction is felt rotor upstream and its typical intensity is of the order of a 1$2 \%$ of the inlet total pressure. Conversely, rotor downstream, the passing of the rotor wakes is a continuous source of injection of turbulence and non-deterministic scales. The forced contribution has been removed, so just small-scale associated to the rotor wake shear layers and instabilities of separated points or wake shedding is taken into account. Higher background levels of $2000 \mathrm{~Hz}$ are observed now at midspan, because of the contribution of the rotor wakes passing by. These are similar values to those found in the stator wakes, though the larger distance of the measuring plane $(50 \mathrm{~mm})$ to the rotor trailing edge with respect to the corresponding plane at the vanes exit $(15 \mathrm{~mm})$ leads to a minor measured value. Both stator wakes and flow separation observed in the interrow spacing are convected through the rotor passage, appearing not fully-mixed at the rotor exit. As the distance from the source has been increased, the frequency is reduced because the integral length scales are further enlarged [21]: the small-scale turbulence is progressively dissipated and only large scales associated to primary flow patterns are preserved [59]. At the hub, important flow separation (also reported in [36]) provokes a further decrease of the cut-off frequency, swept all along the circumferential coordinate with the angular displacement of the blade-to-blade gradients. Anyway, values at the hub boundary layer are similar to those measured rotor upstream.

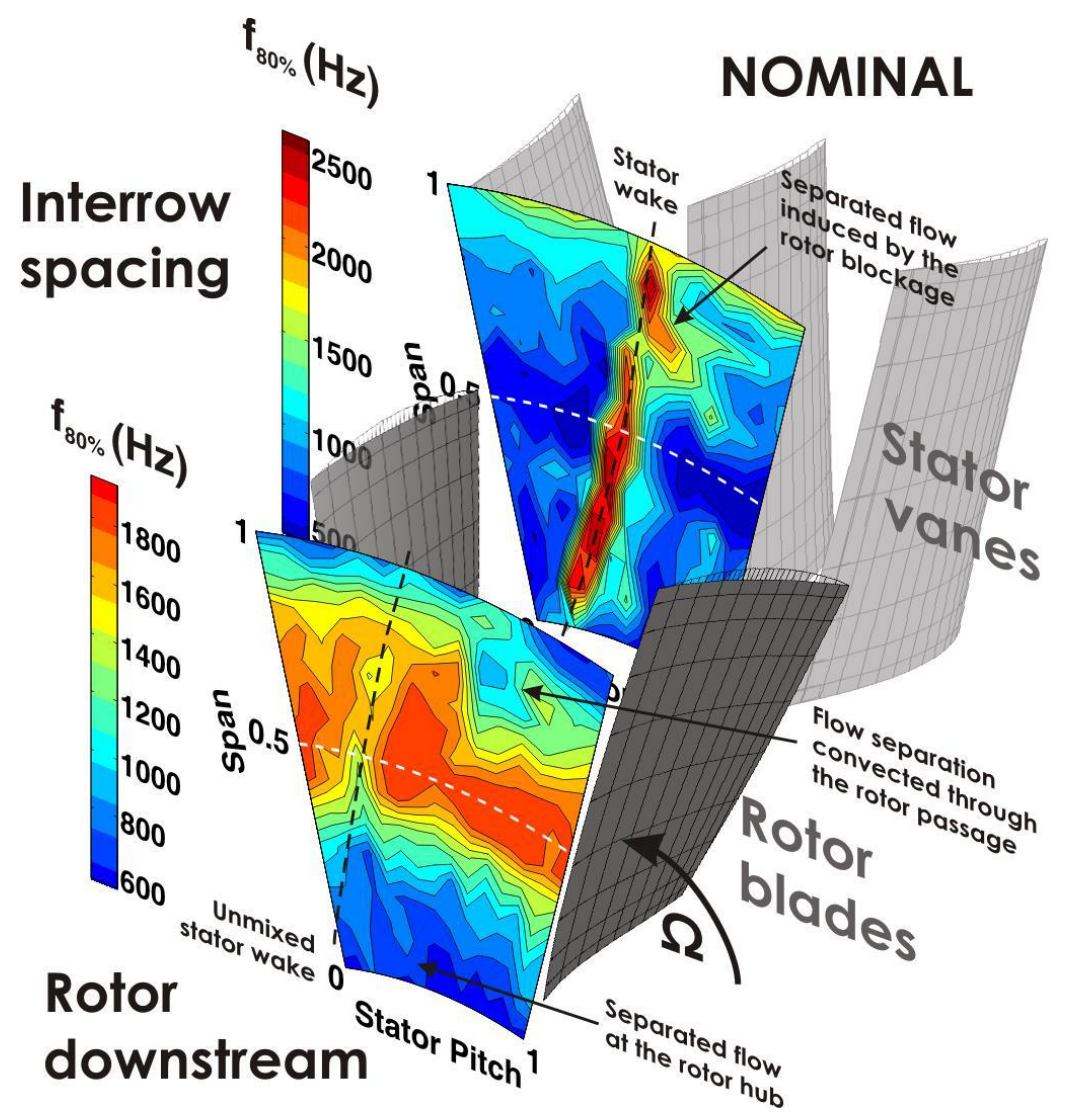

Figure 6. Distributions of the cut-off frequencies corresponding to an $80 \%$ of the cumulative nondeterministic kinetic energy at nominal conditions in the interrow spacing and rotor downstream. 
To reduce data, the maps shown in figure 6 are pitch-averaged (circumferentially) in order to obtain radial distributions of frequencies including $80 \%$ of the nondeterministic energy. A comparison of the different operating points is evaluated in figure 7. Between the rows, the contribution of the stator wakes is smeared out in the averaging, while maximum values are observed in the tip region because of the pitchwise contribution of the boundary layer. However, no remarkable differences are observed with variations in the operating point of the axial fan, as expected from the independency of the stator flow with the mass flow rate [36]. On the contrary, rotor downstream there is a reduction at midspan locations in the maximum frequency when the mass flow rate is decreased. This unexpected trend can be related to the growth of the rotor wakes at off-design conditions, balancing part of the total unsteady energy to the forced component and thus decreasing the small-scale contribution.

These characteristic values of the cut-off frequency for an $80 \%$ of the non-deterministic energy may help to estimate the spatial resolution needed in an accurate LES modelling. It is demonstrated [60] that $80 \%$ of non-deterministic kinetic energy can be resolved introducing a LES filter of size $\Delta \approx 0.19 L_{11}$, where $L_{11}$ represents the longitudinal length scale of largest eddies. A grid size according to this LES filter may resolve the largest eddies within 8 cells [48]. To estimate the integral length scale, the cumulative turbulent kinetic energy as a function of the ratio between the corresponding eddy size and $L_{11}$ is employed. Hence, for high Reynolds number, it can be estimated $L_{11} \approx 1_{80 \%} / 0.42$ where $1_{80 \%}$ stands for the eddy size related to an $80 \%$ of the cumulative kinetic energy [60]. Since in spatial spectrum, the eddies wavenumber is related to the characteristic eddy size according to $\kappa: 2 \pi / 1$ [61], in time spectra we can derive a similar relation, $\omega: 2 \pi U / 1$ where $U$ represents the streamwise velocity responsible for the eddies convection (note that what we obtain measuring in a point with a fixed probe is a time spectra). A rough estimation of the eddies size corresponding to a cumulative energy of $80 \%$ is therefore obtained following $1_{80 \%}=U / f_{80 \%}$. Finally, combining all the above expressions, it is possible to provide recommended values of the mesh density (or LES filter, $\Delta$ ) required to develop accurate simulations.

The table included in figure 7 provides mesh estimations for different zones of the stage, even reporting the influence of the operating conditions. Although these values can be considered only as a crude guideline, they can give an initial hint for early stages when meshing the model. The values obtained for both hub and tip regions are not fully considering the boundary layers, because as we move towards the endwall, the integral length scale tends to zero and an extremely-high dense mesh is required. Only the effect of the outer zones of the boundary layer is applicable here (and flow separations observed in both tip and hub regions in figure 6). In the interrow spacing, both wake and tip regions need a mesh size in the order of $1 \mathrm{~mm}$, whereas at midpassage, a spatial discretization of $5 \mathrm{~mm}$ is sufficient. Rotor downstream, the periodic passing of wakes reduces the integral length scale and, as a consequence, a lower value of $2.7 \mathrm{~mm}$ is now required. As the measuring plane at the rotor exit is farther than the one behind the stator, it is reasonable to obtain a coarser mesh in the vicinity of the rotor wakes than vanes downstream. 
Interrow spacing

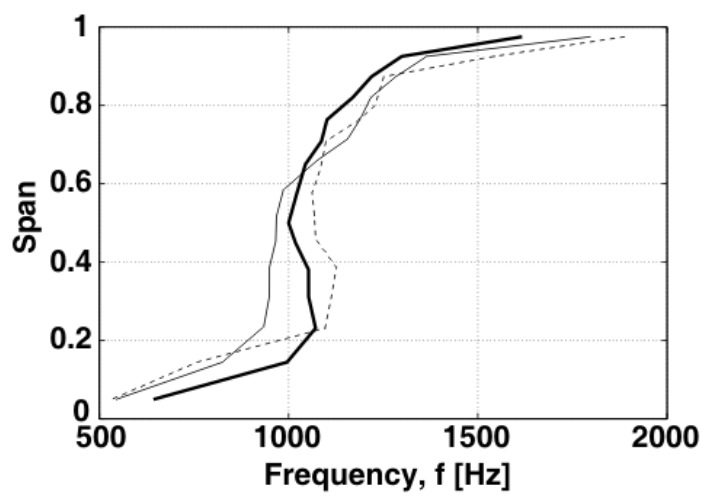

\begin{tabular}{lccc} 
& Nominal & $\begin{array}{c}\text { Partial } \\
\text { Load }\end{array}$ & $\begin{array}{c}\text { Near } \\
\text { Stall }\end{array}$ \\
\hline Tip boundary layer & 1.25 & 0.93 & 0.72 \\
Wake & 1.38 & 1.31 & 1.26 \\
Passage & 9.9 & 7.5 & 5.8 \\
Hub boundary layer & 8.0 & 6.5 & 5.6 \\
\hline
\end{tabular}

Typical mesh size $(\mathrm{mm})$ to resolve an $80 \%$ of the non-deterministic kinetic energy in the interrow spacing.

\section{Rotor downstream}

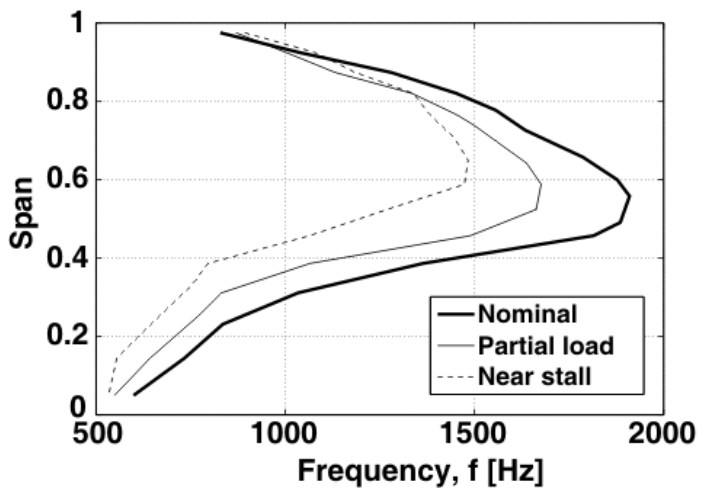

Frequency, $\mathrm{f}[\mathrm{Hz}]$

\begin{tabular}{lccc} 
& Nominal & $\begin{array}{c}\text { Partial } \\
\text { Load }\end{array}$ & $\begin{array}{c}\text { Near } \\
\text { Stall }\end{array}$ \\
\hline Tip boundary layer & 6.1 & 4.8 & 3.9 \\
Wake & - & - & - \\
Passage & 2.6 & 2.7 & 2.9 \\
Hub boundary layer & 7.2 & 6.4 & 5.7 \\
\hline
\end{tabular}

Typical mesh size $(\mathrm{mm})$ to resolve an $80 \%$ of the non-deterministic kinetic energy rotor downstream.

Figure 7. Cut-off frequencies for an $80 \%$ of the non-deterministic energy and estimation of the mesh size required to resolve these scales within a low-speed axial fan stage.

\section{Total Unsteadiness}

Prior to the analysis of the energetic relevance of the unforced contributors, we introduce a glimpse at the features of the total unsteady kinetic energy developed in this axial turbomachine. Briefly, the interest is focused on the identification of the main flow structures and the definition of a significant metric to perform an accurate description. In every point in space, the total kinetic energy of the unsteady part is computed according to $k_{T}=\frac{3}{4}\left(u_{i}^{\prime} u_{i}^{\prime}\right)$ where the coefficient $\frac{3}{4}$ in $k$ is introduced to account for the variance of the out-of-plane velocity component (see [53] for good review of the estimation of radial velocities as the average value of the in-planes variances assuming local isotropy). The distributions obtained in the interrow spacing and rotor downstream for partial load conditions are shown in figure 8.a, made non-dimensional with the overall mean velocity, according to the flow rate, in each measuring sector. Between the rows, the blockage effect of the rotor is observed in the circumferential band from midspan to tip regions, increasing the background level of disturbance from approx. $2 \%$ to roughly a $4 \%$ (specially critical in this case of reduced axial gap). Also, the separated flow previously described close to the vane's suction side is also noticeable in this variable. At rotor downstream, the periodic contribution of the rotor wake deficit is time-averaged in this representation, so the wake-core structure of the rotor flow is pitch-wise redistributed. Important vortical patterns, as the flow separation at the hub or the transport and mixing-out of the tip separation are also recovered in the plot.

To observe the impact of operational parameters, a pitch-averaged reduction of these maps has been also included in plots 8.b. As expected, both situations of reduced gap and off-design working points lead to a notable increase of the total unsteadiness. Notice that the most relevant characteristic is the severe unsteadiness felt in the interrow 
spacing due to the rotor blockage. On the contrary, rotor downstream, these interaction effects are mixed-out, while the important flow separations at the hub become the major source of unsteadiness. As expected, at the rotor exit, the influence of the axial gap distance is only marginal.
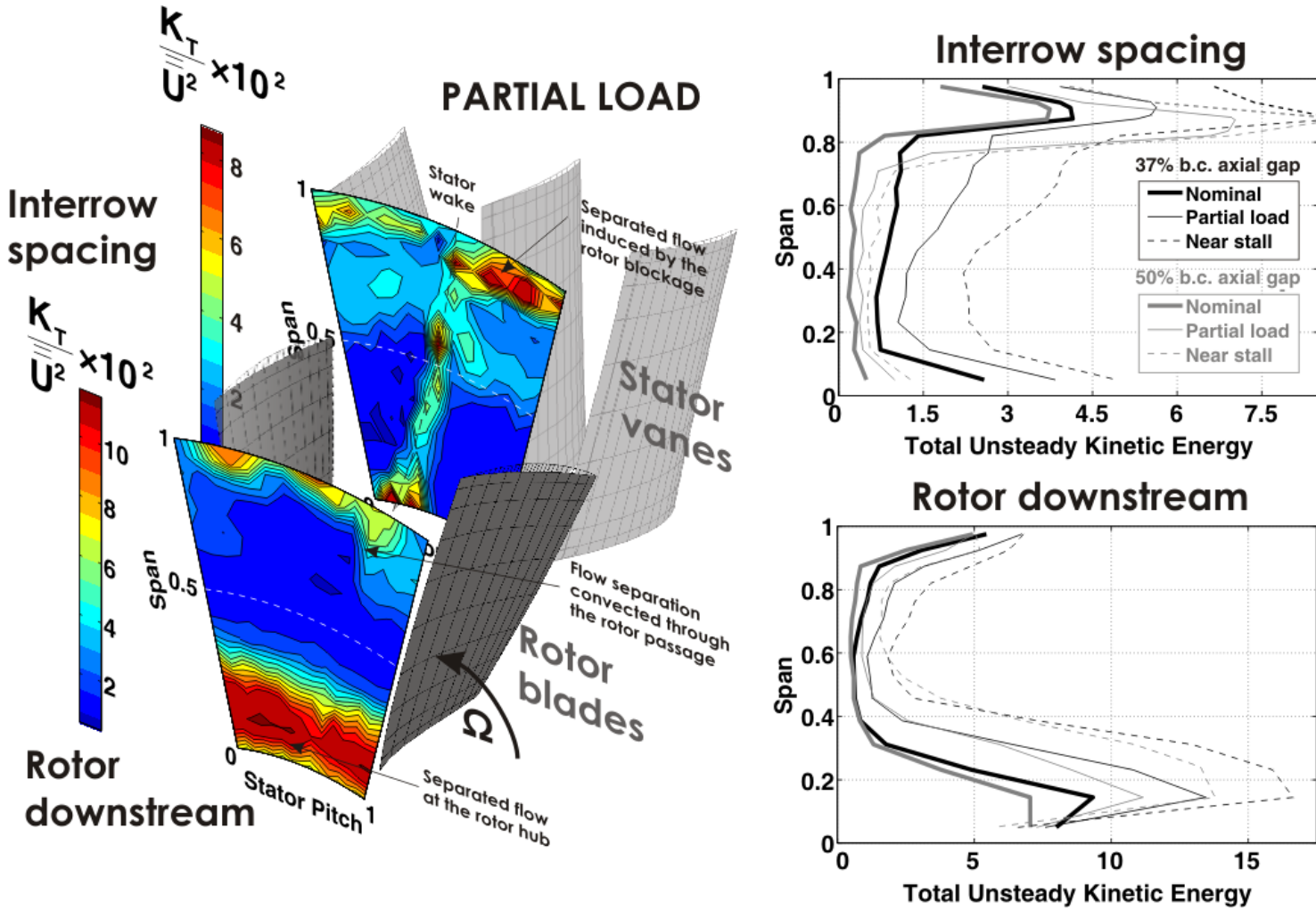

Figure 8. a) Maps of kinetic energy of the total unsteadiness in the interrow spacing and rotor downstream for partial load conditions. b) Comparison of pitch-averaged distributions for different operating conditions.

\section{Unforced unsteadiness}

The significance of all the contributors to the total unsteadiness in the present database is considered hereafter. As previously discussed, the energy associated to the total unsteadiness has been split in three different parts: forced and unforced unsteadiness, mainly related to medium and large scales of the flow, and turbulence, typically linked to the smallest scales of the flow. To provide a simple indicator, the relative weight of each component has been drawn in figure 9 for nominal conditions in terms of percentage respect to the total unsteady kinetic energy. These energy contents have been calculated as the area under the power spectrum density of the velocity magnitude for each component (figures 4 and 5).

Primary flow structures can be identified in all the unsteady components, as a consequence of the progressive energy cascade: large eddies in the order of the passage flow structures, like the wake deficit or the displacement thickness of end-wall boundary layers, are breaking up into smaller ones, like those shed by the turbulent shear layers. Even so, the meaning behind every one is rather different: while the forced unsteadiness reveals the strength of the blade-to-blade mechanisms, responsible for the momentum transfer in the stage, the non-deterministic components give an idea of the disorder and overall disturbance in the flow structure. The left plot shows that deterministic patterns are predominant at midspan: as the rotor sweeps tangentially the 
measuring window, a circumferential band of nearly a $60 \%$ of the total unsteady energy is established in the fixed reference frame. However, the tangential uniformity is broken up by the presence of the unmixed stator wake, pointing out that wake-wake interactions reduce the forced component and tend to increase the non-deterministic levels. Towards tip and hub boundary layers, the forced unsteadiness is significantly reduced (only a negligible $5-10 \%$ is remaining) as a consequence of the loss of the wake-core structure. Concerning unforced unsteadiness, the central map shows that it is practically marginal in the locations where the forced component prevails. Nevertheless, in the zone affected by the wake-wake interaction, the levels are increased up to a relevant 30\%: the stator wake fluid perturbs the vortical structures of the rotor wake, provoking wake oscillations [36] and vortex shedding of separated flows generated at the trailing edge of the blades. This phenomenon is induced by the periodic passing of the rotor (deterministic), so part of its energy (around 20-25\%) is manifested as a forced mechanism. But additionally, the instabilities associated to the interference of the shear layers lead to a dispersed energy spectrum, introducing unforced high-order periodicities (approx. 30\%) and more turbulence (45-50\%). Notice that the tip flow separation (developed at the stator exit in this case with the lower axial gap and convected through the rotor passage) is also contributing, with similar vortical instabilities, to generate unforced scales. Finally, the turbulent scales are the most important component in the end-wall boundary layers, with typical values reaching up a $65 \%$. Furthermore, the interaction phenomena also participate with high turbulence levels. At midspan locations, a background level around 10-15\% reports the passageaveraged footprint of the turbulent scales within the rotor wakes.

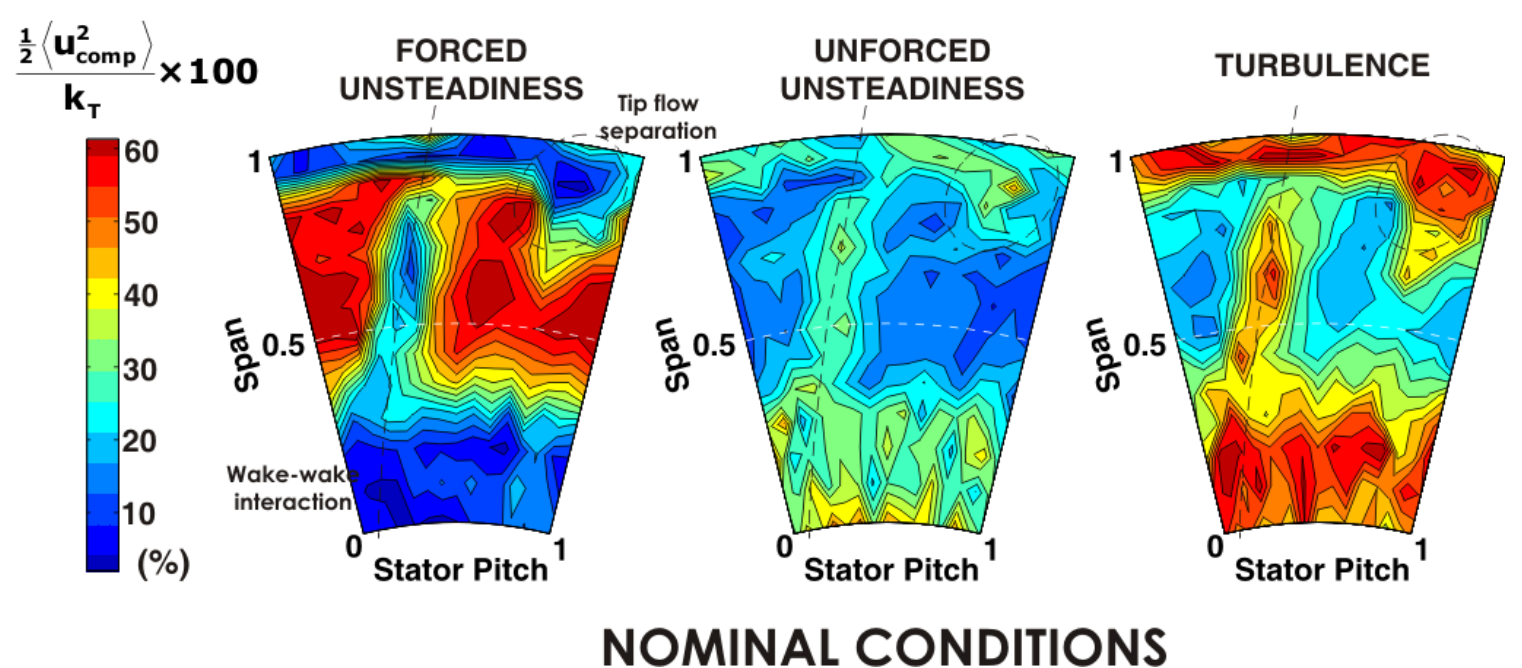

Figure 9. Relative contribution of deterministic and non-deterministic components to the total unsteady kinetic energy in the vicinity of the rotor wake at nominal conditions.

Figures 10 and 11 show the influence of the operating conditions on the distributions of the energy components. As expected, as the flow rate is reduced, the progressive degradation of the deterministic patterns lead to major flow disorders and a massive presence of turbulent structures. At off-design conditions, the hub boundary layer is enlarged and high levels of forced unsteadiness are notably reduced, because of the thickening of wake-wake interaction [36-37] and the massive blockage in the tip. Nondeterministic scales are thus reinforced, particularly evident in the generation of 
turbulence at both hub and tip sections. Similarly, an enlargement of the unforced affection is experienced, rather than a real level increase. Hence, characteristic values of a 20-30\% are observed elsewhere for the unforced component. Only in the hub boundary layer are further increased, though no definitive conclusions can be drawn there: the complexity of the three-dimensional flow developed in the end-wall boundary layers suggest us to consider these values only as representative of overall trends.

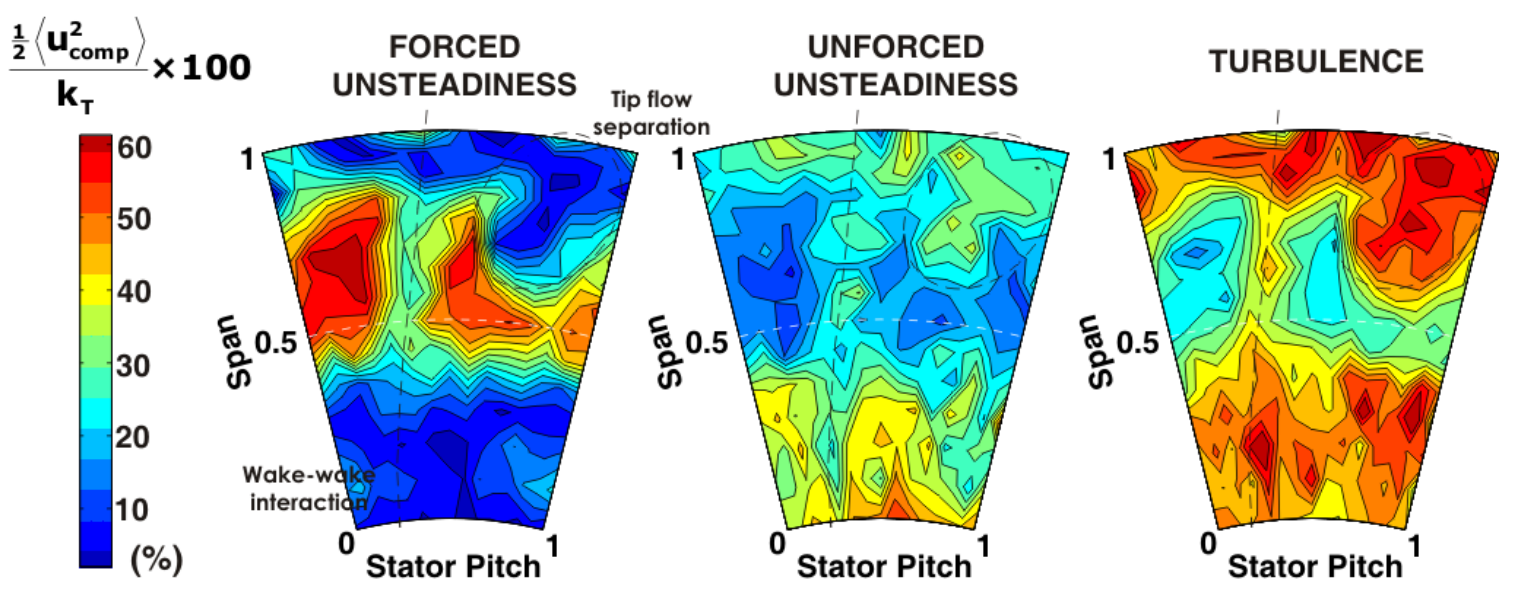

PARTIAL LOAD

Figure 10. Relative contribution of deterministic and non-deterministic components to the total unsteady kinetic energy in the vicinity of the rotor wake at partial load.

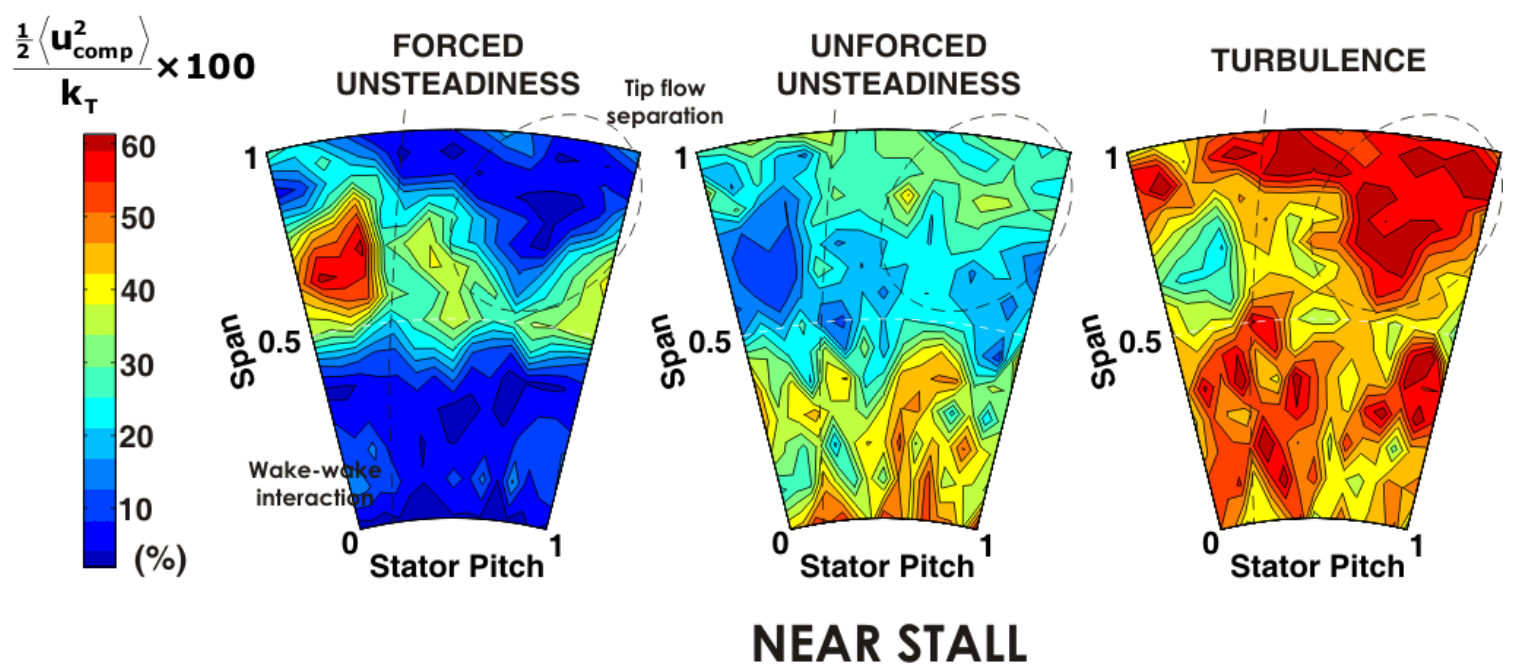

Figure 11. Relative contribution of deterministic and non-deterministic components to the total unsteady kinetic energy in the vicinity of the rotor wake near stall.

The radial distribution of the different components contributing to the total unsteadiness at midspan is now presented in figure 12 for all the operating conditions. These results have been obtained pitch-averaging the planar maps discussed above. The representation is a bar graph of the averaged total unsteadiness (i.e., its associated kinetic energy) which includes the relative contribution (in percentage as before) of each component at every radial position of the database. For every bar, the black part corresponds to the turbulent kinetic energy whereas white and gray parts represent both forced and unforced components, respectively. A moderate non-dimensional scale 
ranging from 0 to 15 has been employed here, in a similar fashion to the colorbars of fig. 8. Then, only midspan positions are now considered because the total unsteady kinetic energy shoots up close to the end-walls. More comments regarding the energy budget of the unforced component in the tip region will be introduced later.

Basically, it is observed a progressive enlargement of the total unsteadiness for offdesign conditions, as well as a significant increase in the contribution of turbulent scales: from roughly a $30 \%$ at design operation to nearly $50 \%$ near stall. As already mentioned, the forced structures are balanced towards the non-deterministic contributions (mainly turbulence), going from a $60 \%$ at nominal conditions to just a $35 \%$ for the lowest flow rate. On the contrary, the unforced component remains practically constant, in the order of a $17-19 \%$ whatever flow rate considered. This finding is in consonance with previous observations of the authors for this type of industrial axial fans: while both turbulent and forced mechanisms show important quantitative variations with the operating conditions, the unforced component is practically independent to this parameter [40]. Along the radial coordinate, as we move away from central positions, the forced contributions are also continuously reduced and much of the unsteady energy is again transferred to the turbulent scales.

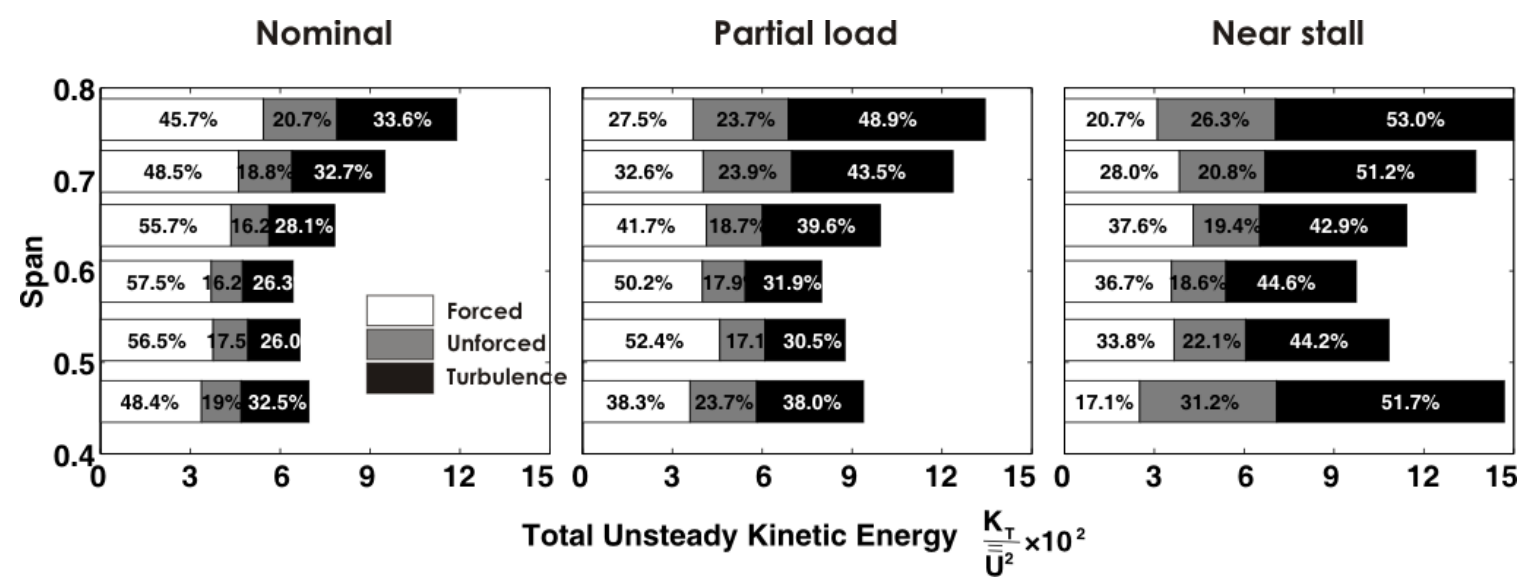

Figure 12. Radial distributions of pitch-averaged unforced unsteadiness at midspan locations.

The frequential segregation of the unforced component allows us to obtain a "fictitious" temporal series when its power spectrum is transformed back into the time domain. This inverse transformation is executed scaling the amplitudes of the raw data and then coupling these values with the phase information of every wavenumber coming from the original series. Afterwards, this component can be further ensemble-averaged to get valuable information about the transport of these unsteady structures in the deterministic flow. In essence, the filtering technique defined for this set of experiments has provided an unforced time-signal (cleaned off deterministic periodicities and turbulence) that can be even reduced to one blade passage. Thus, it is possible to observe if the distribution of unforced unsteadiness is spatially linked to the flow structures of the rotor passage. To do so, the phase-averaged distributions need to be referred to the relative reference frame, using an algorithm that relocates in both phase and time the complete velocity set of each test [38]. As a consequence, if maximum values of unforced unsteadiness are encountered in the tip vortex region of the rotor passage, we may conclude that part of the large-scale unsteadiness has to be locked to the tip leakage flow. 
Figure 13 shows the result of this procedure for nominal conditions when the interrow spacing is the largest (the three-dimensional view). The kinetic energy associated to the unforced component is made non-dimensional with the kinetic energy of the bulk flow, as usual. Now, the spatial distribution covers the rotor pitch so we can observe the rotor wakes and all the relevant structures associated to the rotating passages. This representation illustrates that the unforced energy is linked to the rotor sources, as demonstrated from the appearance of the rotor wakes. Moreover, an intense core of unsteadiness in the tip region indicates the contribution of tip vortex structures onto the unforced component. At the hub, there is also a remarkable presence of unforced phenomena, which could indicate the presence of passage vortices. In the right column of the figure, similar conclusions can be derived when analyzing off-design situations, reporting a progressive growth of the unforced scales in the vicinity of the rotor wake and also in the tip leakage flow.
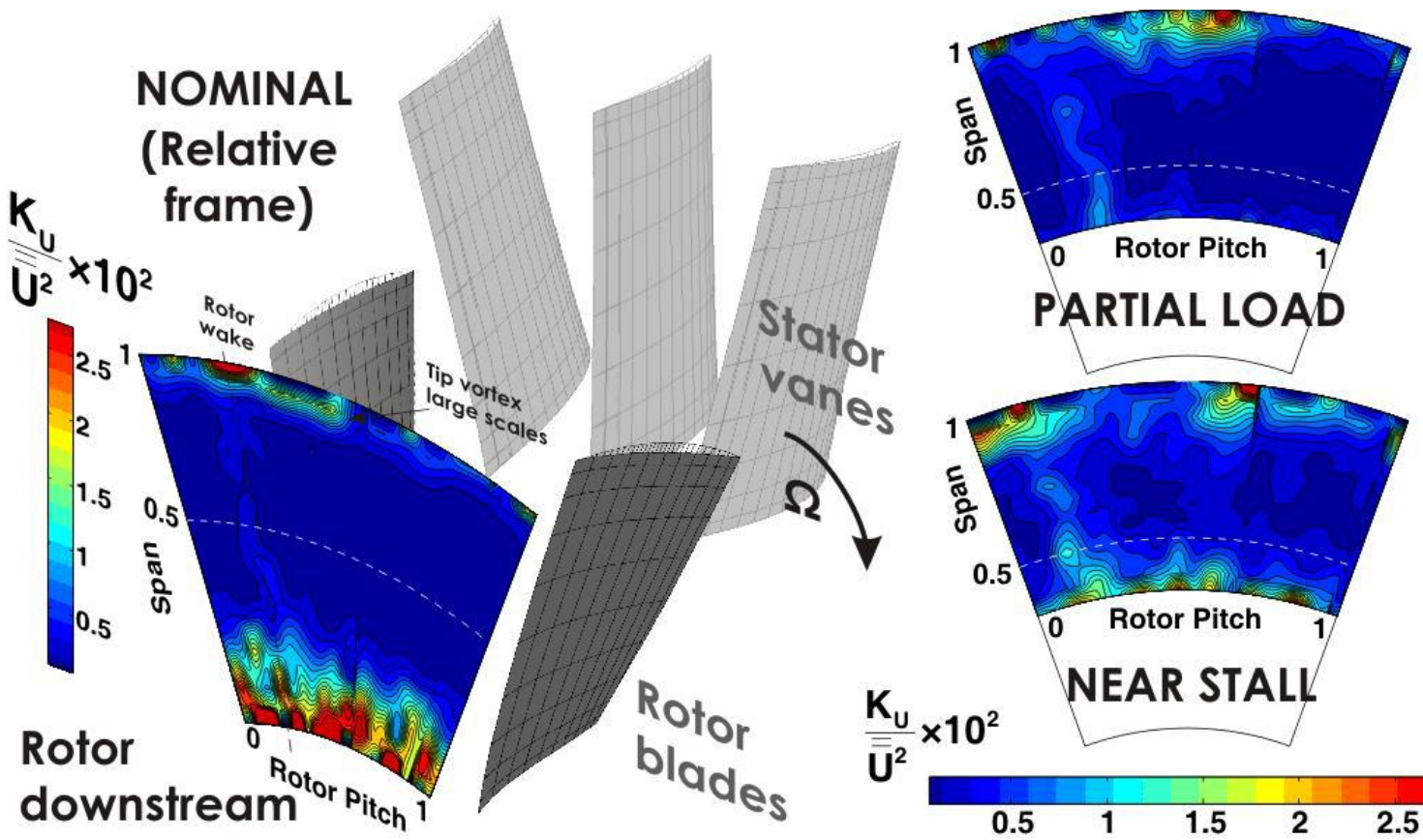

Figure 13. Distributions of forced unsteadiness in the relative frame of reference, downstream of the rotor for different operating conditions.

\section{Conclusions}

The present investigation deals with large-scale unsteadiness that appears superimposed to the turbulent scales in the energy spectrum of the non-deterministic flow in lowspeed axial fans. This unforced component, non-related with the deterministic contributions at the BPF rate, is a consequence of other periodic mechanisms like unstable separation points, wake oscillations, instabilities on the tip vortex or even blade misalignments. Usually masked as large-scale eddies of random turbulence, this component requires of a frequency-based procedure to isolate its contribution within the non-deterministic part of the total unsteadiness.

The methodology presented here is applied over a complete experimental database obtained using dual hot-wire anemometry within the stage of the fan. The experiments are performed in an open-loop facility that allows the variation of the axial gap between 
the rows and the regulation of the operating conditions. In recent years, the data obtained has been carefully analysed by the authors, so valuable information concerning interaction phenomena and unsteady energy budgets in the vicinity of rotor wakes has been provided to the literature. All the experience gained in these investigations has encouraged us to keep focused on the diverse features of the different time and length scales involved in turbomachinery flows.

The identification of the unforced component requires two steps. Firstly, the phaselocked averaging technique is introduced to segregate the time-resolved fluctuations of the flow from the raw velocity traces. Afterwards, a parametric estimation of the baseline spectrum of turbulence and an iterative procedure to filter relevant peaks in the smoothed periodogram is used to isolate the unforced contributions. A detailed analysis of the characteristic time scales associated to the unforced component has revealed that most part of its energy is redistributed within the frequency range containing an $80 \%$ of the total unsteady kinetic energy. Since LES simulations are typically conceived to resolve that cumulative energy level within the fixed filter, it is expected that accurate models with sufficient spatial discretization may address the unforced scales in the resolution. On the contrary, unsteady RANS are susceptible to require further modelling to take them into account. In addition, the cut-off frequencies containing that $80 \%$ of the total unsteadiness have been employed to estimate the order of the mesh size required in the rotor passage and the interaction zones to achieve a well-resolved LES modelling. Note that close to the end-walls and blade surfaces, the LES description of the boundary layers needs much higher resolution, so only typical mesh sizes of interior regions are suggested here (guidelines for near-wall meshing are beyond the scope of this paper).

As a starting point, levels of total unsteadiness within the stage have been illustrated, with special interest on the structures observed rotor downstream. The significance of the three unsteady components -forced and unforced unsteadiness and turbulence- has been done in terms of percentage with respect to the total unsteady energy. Complete maps in transversal planes have shown that primary flow structures can be identified in all the unsteady components due to the redistribution of all-range scales throughout the energy cascade. Also, the influence of the operating conditions and the axial gap between the rows on these components has been considered in the investigation. Forced mechanisms are shown to be predominant at design conditions, especially at midspan, with more than $60 \%$ of the total energy. As the mass flow rate is reduced, the turbulent structures are enhanced as a consequence of a major flow disorder and the thickening of the casing boundary layers. Additionally, wake-wake interactions appear as an important source of unforced unsteadiness, contributing with approximately a $20 \%$ of the total energy in central positions, though these levels are practically independent to changes in the flow rate. Pitch-averaged distributions at midspan smear the impact of the unmixed stator wakes, but confirm global trends in the balance of forced unsteadiness to turbulent scales as the fan operates at off-design conditions. Towards tip and hub regions, the total unsteadiness is severely increased so the complex threedimensionality of the flow and the high generation of turbulence tend to mask the unforced scales. However, maps of averaged unforced unsteadiness in the relative reference frame have revealed that instabilities in the tip leakage flow are addressed as large-scale phenomena.

Future work must be focused on the development of a model to describe the transport and mixing-out of unforced scales. Complementarily, the numerical modelling of this low-speed axial fan using both DES and URANS turbulent closures will highlight differences in the estimation of turbulence levels when the contribution of unforced scales is neglected. 


\section{Acknowledgements}

This work was supported by the Research Project "Characterization of the Generation of Aerodynamic Noise due to the Interaction between the Rotor Blades and the Guide Vanes in Axial Flow Fans", DPI2006-15720, CICYT.

\section{References}

[1] Abhari, R.S., 2006, Unsteady fluid dynamics of turbines: a perspective on possible directions to improve future engine designs. Proceedings of Minnowbrook V. Workshop on Unsteady Flows in Turbomachinery, Blue Mountain Lake, NY, 20-23 August, 2006.

[2] Adamczyk, J.J., 1996, Modelling the effect of unsteady flows on the time average flow field of a blade row embedded in an axial flow multistage turbomachine. VKI Lecture series, 1996-05.

[3] Chow, Y.-C., Uzol, O., Katz, J., Meneveau, C., 2005, Decomposition of the spatially filtered and ensemble averaged kinetic energy, the associated fluxes and scaling trends in a rotor wake. Physics of Fluids, 17, 085102.

[4] Sharma, O.P., Stetson, G.M., Danields, W.A., Greitzer, E.M., Blair, M.F., Dring, R.P., 1997, Impact of periodic unsteadiness on performance and heat load in axial flow turbomachines. NASACR-202319, 1997.

[5] Van de Wall, A.G., Kadambi, J.R., Adamczyk, J.J., 2000, A transport for the deterministic stresses associated with turbomachinery blade row interactions. ASME Journal of Turbomachinery, 122, 593-603.

[6] Dring, R.P., Joselyn, H.D., Hardin, L.W., Wagner, J.H., 1982, Turbine rotor-stator interaction. ASME Journal of Engineering for Power, 104, 729-742.

[7] Adamczyk, J.J., 2000, Aerodynamic analysis of multistage turbomachinery flow in support of aerodynamic design. ASME Journal of Turbomachinery, 122, 189-217.

[8] Leboeuf, F., 2002, Unsteady flow analysis in transonic turbines and compressor stages. VKI Lecture series, 2002-01.

[9] Uzol, O., Chow, Y.-C., Katz, J., Meneveau, C., 2002, Experimental investigation of unsteady flow field within a two-stage axial turbomachine using particle image velocimetry. ASME Journal of Turbomachinery, 124, 542-552.

[10] Lakshminarayana, B., 1995, Fluid Dynamics and Heat Transfer of Turbomachinery. Ed. John Wiley and Sons.

[11] Dean, R. C., 1959, On the necessity of unsteady flow in fluid machines. ASME Journal of Basic Engineering, 81, 24-28.

[12] Greitzer, E.M., Tan, C.S., Graf, M.B., 2004, Internal Flow: Concepts and Applications. Cambridge University Presss.

[13] Lyman, F.A., 1993, On the conservation of rothalpy in turbomachines. ASME Journal of Turbomachinery, 115, 520-526.

[14] Van Zante, D.E., Adamczyk, J.J., Strasizar, A.J., Okiishi, T.H., 2002, Wake recovery performance benefit in a high-speed axial compressor. ASME Journal of Turbomachinery, 124, 275-284.

[15] Prato, J., Lakshminarayana, B., Suryavamshi, 2001, Three-dimensional flow field downstream of an embedded stator in a multistage axial flow compressor. Part 1: steady and unsteady flow fields. Proceedings of the Institution of Mechanical Engineers, Part A: Journal of Power and Energy, 215, 281-299

[16] Jang, C.M., Sato, D., Fukano, T., 2005, Experimental analysis on tip leakage and wake flow in an axial flow fan according to flow rate. ASME Journal of Fluids Engineering, 127, 322-329.

[17] Dénos R., Paniagua G., 2005, Effect of vane-rotor interaction on the unsteady flowfield downstream of a transonic HP turbine". Proceedings of the Institution of Mechanical Engineers, Part A: Journal of Power and Energy, 219, 431-442.

[18] Henderson, A.D., Walker, G., Hughes, J.D., 2006, Unsteady transition phenomena at a compressor blade leading edge. ASME Paper GT2006-90641, Proceedings of the ASME TurboExpo, Barcelona, Spain, 8-11 May, 2006.

[19] Mailach, R., Vogeler, K., 2004, Aerodynamic blade row interactions in an axial compressor - Part I: unsteady boundary layer development. ASME Journal of Turbomachinery, 126, 35-44.

[20] Hodson, H.P., Howell, R.J., 2005, Bladerow interactions, transition and high-lift aerofoils in low- 
pressure turbines. Annual Review of Fluid Mechanics, 37, 71-98.

[21] Henderson, A.D., Walker, G., Hughes, J.D., 2006, The influence of turbulence on wake dispersion and blade row interaction in an axial compressor. ASME Journal of Turbomachinery, 152, 150157.

[22] Chow, Y.-C., Uzol, O., Katz, J., 2002, Flow nonuniformities and turbulent hot spots due to wakeblade and wake-wake interactions in a multi-stage turbomachine. ASME Journal of Turbomachinery, 124, 553-563.

[23] Lakshminarayana, B., 1981, Techniques for aerodynamic and turbulence measurements in turbomachinery rotors. ASME Journal of Engineering and Power, 103, 374-392.

[24] Tropea, C., Yarin, A.L., Foss, J.F. (Eds.), 2008, Handbook of Experimental Fluid Mechanics. Springer.

[25] Cherret, M.A., Bryce, J.D., 1992, Unsteady viscous flow in high-speed core compressor. ASME Journal of Turbomachinery, 114, 287-294.

[26] Read, S., Elder, R.L., 1999, The nature of wakes in multistage axial flow compressors. Proceedings of the Institution of Mechanical Engineers, Part A: Journal of Power and Energy, 213, 275-289.

[27] Witkowski, A.S., Chmielniak, T.J., Strozik, M.D., 1996, Experimental study of a 3D wake decay and secondary flows behind a rotor blade row of a low speed axial compressor. ASME Paper 96GT-415, Proceedings of the ASME TurboExpo, Birmingham, England, 10-13 June, 1996.

[28] Sentker, A., Reiss, W., 2000, Experimental investigation of turbulent wake-blade interaction in axial compressors. International Journal of Heat and Fluid Flow, 21, 285-290.

[29] Sinha, M., Katz, J., 2000, Quantitative visualization of the flow in a centrifugal pump with diffuser vanes-Part I: On flow structures and turbulence. ASME Journal of Fluids Engineering, 122, 97107.

[30] Suder, K.L., Hathaway, M.D., Okiishi, T.H., Strazisar, A.J., Adamczyk, J.J., 1987, Measurements of the unsteady flow field within the stator row of a transonic axial-flow fan. NASA TM-88945, 1987.

[31] Velarde-Suárez, S., Ballesteros-Tajadura, R., Santolaria-Morros, C., Blanco Marigorta, E., 2002, Total unsteadiness downstream o fan axial flow fan with variable pitch blades. ASME Journal of Fluids Engineering, 124, 280-283.

[32] Ballesteros, R., Velarde, S., Santolaria, C., 2002, Turbulence intensity measurements in a forwardcurved blades centrifugal fan. Proceedings of the 21st IAHR Symposium in Hydraulic Machinery and Systems, Laussane, Switzerland, 9-12 September, 2002.

[33] Fernández Oro, J.M., Argüelles Díaz, K.M., Ballesteros Tajadura, R., Santolaria Morros, C., 2002, Numerical analysis of the rotor-stator interaction in an axial flow blower. Proceedings of the 21st IAHR Symposium in Hydraulic Machinery and Systems, Laussane, Switzerland, 9-12 September, 2002.

[34] Fernández Oro, J.M., Argüelles Díaz, K.M., Santolaria Morros, C., Ballesteros Tajadura, R., 2003, Unsteady flow analysis of the stator-rotor interaction in an axial flow fan. Proceedings of the 4th ASME-JSME Joint Fluids Engineering Summer Conference, Honolulu, HW, 6-10 July, 2003.

[35] Fernández Oro, J.M., Argüelles Díaz, K.M., Santolaria Morros, C., Blanco Marigorta, E., 2005, Unsteady rotor-stator interaction in an axial flow blower. Part I: Numerical and experimental flow field characterization - Part II: Deterministic stresses analysis. Proceedings of the 2005 Fluids Engineering Division Summer Meeting, Houston, TX, 19-23 June, 2005.

[36] Fernández Oro, J.M., Argüelles Díaz, K.M., Santolaria Morros, C., Blanco Marigorta, E., 2007, Unsteady flow and wake transport in a low-speed axial fan with inlet guide vanes. ASME Journal of Fluids Engineering, 129, 1015-1029.

[37] Fernández Oro, J.M., Argüelles Díaz, K.M., Santolaria Morros, C., Blanco Marigorta, E., 2007, On the structure of turbulence in a low-speed axial fan with inlet guide vanes. Experimental Thermal and Fluid Science, 32(1), 316-331.

[38] Fernández Oro, J.M., Argüelles Díaz, K.M., Santolaria Morros, C., Blanco Marigorta, E., 2008, Analysis of the deterministic unsteady flow in a low-speed axial fan with inlet guide vanes. ASME Journal of Fluids Engineering, 130, 031101.

[39] Fernández Oro, J.M., Ballesteros Tajadura, R., Blanco Marigorta, E., Argüelles Díaz, K.M., Santolaria Morros, C., 2008, Turbulence and secondary flows in an axial flow fan with variable pitch blades. ASME Journal of Fluids Engineering, 130, 041101.

[40] Fernández Oro, J.M., Blanco Marigorta, E., Argüelles Díaz, K.M., Ballesteros Tajadura, R., 2009, Forced and unforced unsteadiness in an axial turbomachine. Experimental Thermal and Fluid Science, in press.

[41] Yao, J., Davis, R.L., Alonso, J.J., Jameson, A., 2002, Massively parallel simulation of the unsteady 
flow in an axial turbine stage. Journal of Propulsion and Power, 18(2), 465-471.

[42] Arnone, A., Marconcini, M., Scotti del Greco, A., Spanio, E., 2004, Numerical investigation of three-dimensional clocking effects in a low pressure turbine. ASME Journal of Turbomachinery, 126, 375-384.

[43] Fernández Oro, J.M., Argüelles Díaz, K.M., Santolaria Morros, C., 2006, Unsteady 3D simulation of a jet fan with symmetric blades. Proceedings of the 2006 Fluids Engineering Division Summer Meeting, Miami, FL, 17-20 July, 2006.

[44] Kirtley. K.R., Beach, T.A., 1992, Deterministic blade row interactions in a centrifugal compressor stage. ASME Journal of Turbomachinery, 114, 304-311.

[45] Rhie, C.M., Gleixner, A.J., Spear, D.A., Fischberg, C.J., Zacharias, R.M., 1998, Development and application of a multistage Navier-Stokes solver. Part I - Multistage modeling using body forces and deterministic stresses. ASME Journal of Turbomachinery, 120, 205-214.

[46] Bardoux, F., Leboeuf, F., 2001, Impact of deterministic correlations on the steady flow field. Proceedings of the Institution of Mechanical Engineers, Part A: Journal of Power and Energy, 215, 687-698.

[47] Charbonnier, D., Leboeuf, F., 2005, Steady flow simulation of rotor-stator interactions with an unsteady deterministic model. Proceedings of the 6th European Conference on Turbomachinery, Euroturbo, Lille, France, 7-11 March, 2005.

[48] Celik, I.B., Cehreli, Z.N., Yavuz, I., 2005, Index of resolution quality for large hedí simulations. ASME Journal of Fluids engineering, 127, 949-958.

[49] Sagaut, P., 2002, LES and DNS simulation of Turbomachinery Flows. VKI Lecture Series, 200201.

[50] Piomelli, U., 2006, Large-eddy simulation of turbulent flows. VKI Lecture Series 2006-04.

[51] Goto, A., 1992, Three-dimensional flow and mixing in an axial flow compressor with different rotor tip clearances, ASME Journal of Turbomachinery, 112, 675-685.

[52] Brunn, H.H., 1995, Hot-wire Anemometry Principles and Signal Analysis. Oxford University Press.

[53] Uzol, O., Brzozowski, D., Chow, Y.-C., Katz, J., Meneveau, C., 2007, A database of PIV measurements within a turbomachinery stage and sample comparisons with unsteady RANS, Journal of Turbulence, $\mathbf{8}(10)$.

[54] Sentker, A., Reiss, W., 1998, Measurement of unsteady flow and turbulence in a low speed axial compressor, Experimental Thermal and Fluid Science, 17, 124-131.

[55] März, J., Hah, C., Neise, W., 2002, An experimental and numerical investigation into the mechanisms of rotating instability, ASME Journal of Turbomachinery, 124, 367-375.

[56] Hayes, M.H., 1996, Statistical Digital Signal Processing and Modeling. Ed. John Wiley \& Sons.

[57] Levalle, J., Ashpis, D.E., 2004, Estimation of time scales in unsteady flows in a turbomachinery rig. NASA TM-2004-209452

[58] Barmpalias, K., Chokani, N., Kalfas, A.I., Abhari, R.S., 2006, Estimation of spectra by a data adaptative spectral method, Proceedings of the 18th Symposium on Measuring Techniques in Transonic and Supersonic Flow in Cascades and Turbomachines, Thessaloniki, Greece, 21-22 September, 2006.

[59] Davidson, P.A., 2004, Turbulence: an introduction for scientists and engineers. Oxford University Press.

[60] Pope, S.B., 2000, Turbulent flows. Cambridge University Press.

[61] Tennekes, H., Lumley, J.L., 1972, A First Course in Turbulence. The MIT Press. 
This document is a pre-print version of the scientific paper published by Taylor \& Francis. It has been released by the authors to fulfill all the publisher requirements established for Article Sharing:

https://authorservices.taylorandfrancis.com/sharing-your-work/

\section{(c) (1) (9)}

(C) 2019. This manuscript version is made available under the Creative Commons Attribution-NonCommercial-NoDerivatives 4.0 International License (CC-BY-NC-ND 4.0 license) http://creativecommons.org/licenses/by-nc-nd/4.0/ 